\title{
Creep motion of a solidification front in a two-dimensional binary alloy
}

\author{
X. Feng, E. A. Brener, D. E. Temkin, Y. Saito, ${ }^{*}$ and H. Müller-Krumbhaar \\ Institut für Festkörperforschung, Forschungszentrum Jülich, D-52425 Jülich, Germany
}

(Received 14 July 1998)

\begin{abstract}
The propagation of a solidification front in a two-dimensional binary alloy is studied by Monte Carlo simulations. A random atomic configuration is quenched and the atoms that prefer to be in the liquid phase act as quenched pinning centers to the advancing solidification front. For a system with large kink formation energy $\epsilon$ and finite system width $N$, we show that the liquidus and solidus lines in the equilibrium phase diagram correspond to pinning-depinning transition lines, like in a one-dimensional system. In the one-phase region the front is depinned and propagates steadily, whereas in the two-phase region it is pinned and the velocity $v$ decays as time $t$ passes with a power-law behavior $v(t) \sim t^{\nu-1}$, with $\nu<1$. For a moderate $\epsilon$ or for a large width $N$, the pinning transition is smeared out and the front propagates steadily even in the two-phase region by thermal creep. When the driving force $H$ is small, the velocity $v$ decays exponentially with $\epsilon$ and $H^{-1}$. The size dependence is interpreted in terms of the height correlation. [S1063-651X(99)06601-5]

PACS number(s): 05.60.Cd, 66.30.Dn, 05.40.+j
\end{abstract}

\section{INTRODUCTION}

Interface motion in random media is observed in various disciplines of physics: random magnets, charge density waves, steps on crystal surfaces, to name just a few examples. It can be generalized to elastic manifolds such as polymers, vortices in superconductors, etc. In these systems, quenched randomness pins the motion of the interface and at absolute zero temperature a sharp pinning-depinning transition takes place at a finite strength of an external driving force. At a finite temperature, however, the pinning transition is smeared out by the creep motion of the interface.

In this paper we study the creep motion of a onedimensional solidification front in a binary alloy system in two dimensions. The simpler problem of a one-dimensional (1D) system with a zero-dimensional interface has been studied by Temkin and co-workers [1-4]. The atomic configuration throughout the whole system is frozen and the system evolves only by the motion of the solid-liquid interface [5]. For this diffusionless model, the equilibrium phase diagram in the phase space of concentration and temperature is found to determine the critical pinning. In a one-phase region, the interface moves steadily and in the two-phase region the steady velocity vanishes. Atomic clusters which prefer to remain in the liquid state act as quenched obstacles to hinder the propagation of the solidification front. This onedimensional system is found to be equivalent to a random hopping model with a power-law distribution of waiting times for interface jumps [6-12]. In the two-phase region the interface position $h(t)$ shows an anomalous time dependence as a power-law behavior $h(t) \sim t^{\nu}$ with an exponent $\nu<1$ which depends on both the temperature and the concentration of the alloy.

In the present work we study the same system but in two dimensions. In the two-dimensional (2D) system the $1 \mathrm{D}$ in-

\footnotetext{
*Permanent address: Dept. of Physics, Keio University, 3-14-1 Hiyoshi, Kohoku-ku, Yokohama 223, Japan.
}

terface extends in the direction normal to its propagating direction. Then a new degree of freedom comes into play: The interface tension tries to keep the interface straight and counteracts the roughening effect by thermal fluctuations and by the quenched randomness. We shall find that the elastic restoring effect due to the interface tension leads to correlations along the interface, but the correlation length grows very slowly with time. For a system with a finite size and a large restoring force, the correlation eventually catches up to the system size and then the interface in the 2D system shows the same asymptotic dynamics as the 1D system. However, for a large system with a small restoring force, the interface propagates steadily even in the two-phase region. An absence of critical pinning in a large 2D system was already shown qualitatively in a Monte Carlo study by Jackson et al. [14]. Here we study the front propagation in the two-phase region more systematically. It is found to be governed by thermal creep [15], furthermore the steadystate velocity $v$ depends on the driving force $H$ as $v \propto \exp \left(-H^{-1}\right)$. The result can be analyzed in terms of the random field Ising (RFI) model [16] or in terms of driven elastic manifolds $[17,18]$.

The structure of our paper is as follows. In Sec. II, we briefly summarize the main results on a 1D model of an interface moving with random jump probabilities. When they are chosen to reproduce a typical two-component phase diagram of an ideal solution, a pinning-depinning transition takes place at the solid-liquid phase boundary. Steady crystal growth occurs in the solid one-phase region, steady melting in the liquid one-phase region, but nonsteady behavior occurs in the two-phase region. In Sec. III the model is extended to two dimensions where the energy cost for the interface deformation is taken into account. Practically we restrict our investigations to models of solid-on-solid (SOS) type, where overhangs on the interface are not allowed. In particular, we also show that the model can be mapped to a random-field Ising (RFI) model with temperature-dependent random fields. The different behavior of the system inside and outside the two-phase coexistence region is emphasized. 


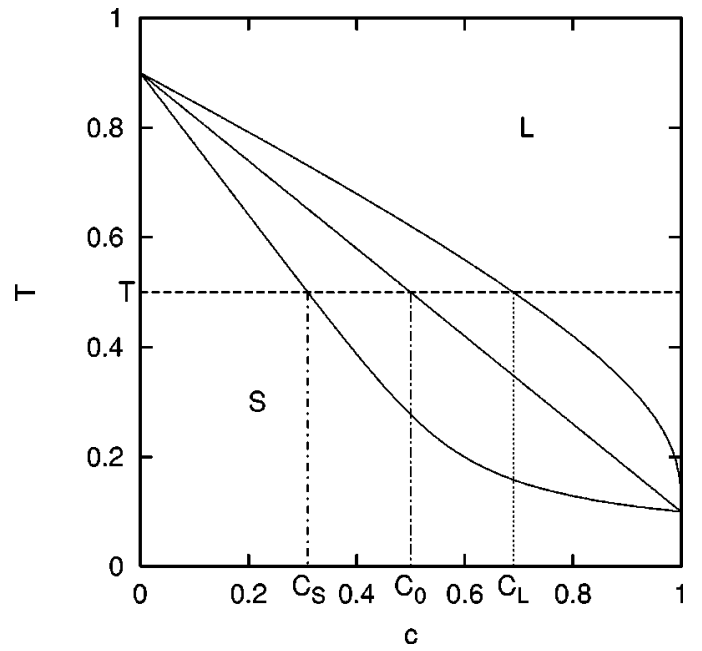

FIG. 1. Equilibrium phase diagram.

In Sec. IV we consider the limit of large bond energies such that the interface between solid and liquid tends to stay very flat on the scale of atomic distances. In this limit we show analytically that the 2D system behaves in many respects like a 1D counterpart with a zero-dimensional interface: The phase boundaries correspond to the pinning-depinning transition lines, and the average interface position in the twophase region shows an anomalous power-law time dependence with exactly the same exponent as in the 1D system. The result is confirmed by detailed MC simulations for the 2D systems with small sizes and large bond energies in Sec. V. But the simulations there also show that a system with an infinite width (i.e., in the limit of infinite length of the onedimensional interface) always settles to a motion of a constant velocity. Even inside the two-phase region, such an infinite system is not pinned but moves at a slow creeping velocity. This creep motion is analyzed in terms of a RFI model, and our numerical results (Secs. V D and VE) confirm quantitatively the analytical estimates of the dependence of the creep velocity upon the driving force and the bond energy. The development of the correlation along the interface explains the finite size and the finite time effects observed in the asymptotic behavior of small systems. Section VI summarizes and concludes our study. Exact but lengthy formulas of transition probabilities and velocities for systems with finite widths and large bond energies are summarized in the Appendix.

\section{SUMMARY OF ONE-DIMENSIONAL RESULTS}

Before we start the study of the 2D system, it is useful for later comparison to briefly summarize the $1 \mathrm{D}$ result. The microscopic model originally proposed by Temkin [1-3] is as follows: On a one-dimensional lattice two species of atoms, $A$ and $B$, are distributed uniformly with the concentration $c$ of $B$ species. This atomic chain is separated by a solid-liquid interface into two parts: the liquid in the upper part and the solid in the lower, for example. The atomic configuration is frozen and atoms cannot move. They can only change their state between the solid and the liquid. $\omega_{+A}$ and $\omega_{-A}$ denote the solidification and melting frequency for an $A$ atom (or probability of phase change per time unit), and
$\omega_{+B}$ and $\omega_{-B}$ denote those for a $B$ atom. The ratio of the probability that an atom $X(=A$ or $B)$ is in the solid state over that for it being in the liquid state is then determined kinetically by $\omega_{-X} / \omega_{+X}$ and this ratio should be equal to the one given thermodynamically as $e^{-\Delta \mu_{X} / T}$, where $\Delta \mu_{X}$ is the chemical potential difference of an atom $X$ between the liquid and solid states. Here and thereafter the temperature $T$ is measured in units of energy. The transition frequencies should then satisfy the relation $\omega_{-X} / \omega_{+X}=e^{-\Delta \mu_{X} / T}$. At a temperature $T$ near the equilibrium melting temperature $T_{X}$ of an $X(=A$ or $B)$ atom, the chemical potential difference is approximately written as $\Delta \mu_{X}=\left(L_{X} / T_{X}\right)\left(T_{X}-T\right)$ with the specific latent heat $L_{X}$. For the mixture of $A$ and $B$ atoms, an ideal solution without mixing energy but with a mixing entropy is assumed. Then the equilibrium phase diagram is obtained with the solidus $C_{s}(T)$ and the liquidus $C_{l}(T)$ lines as

$$
\begin{gathered}
C_{s}(T)=\frac{1-e^{-\Delta \mu_{A} / T}}{e^{-\Delta \mu_{B} / T}-e^{-\Delta \mu_{A} / T}}, \\
C_{l}(T)=\frac{\left(1-e^{-\Delta \mu_{A} / T}\right) e^{-\Delta \mu_{B} / T}}{e^{-\Delta \mu_{B} / T}-e^{-\Delta \mu_{A} / T}},
\end{gathered}
$$

as is shown in Fig. 1.

In the diffusionless phase transition considered here, the solidification proceeds via the propagation of a single solidliquid interface over a frozen configuration of $A$ and $B$ atoms. Although each atom has fixed probability to solidify or melt, the solidification and melting take place only at the interface position. In other words, we exclude the possibility of nucleation of the energetically favorable phase in positions away from the solid-liquid interface. The theoretical analysis by Temkin [1-3] revealed that a steady-state motion of the interface is possible in one-phase regions, but in the two-phase region the average velocity of the interface advancement vanishes with time. In a one-phase region the mean displacement $\langle h(t)\rangle$ of the interface is linear in time $t$ as

$$
\begin{gathered}
\langle h(t)\rangle=v_{+} t \quad\left(c<C_{s}\right), \\
\langle h(t)\rangle=-v_{-} t \quad\left(c>C_{l}\right),
\end{gathered}
$$

with the velocities

$$
\begin{aligned}
v_{+} & =\left(1-\left\langle\frac{\omega_{-}}{\omega_{+}}\right\rangle\right) /\left\langle\frac{1}{\omega_{+}}\right\rangle \\
& =\left\{1-\left[(1-c) \frac{\omega_{-A}}{\omega_{+A}}+c \frac{\omega_{-B}}{\omega_{+B}}\right]\right\}\left[\frac{1-c}{\omega_{+A}}+\frac{c}{\omega_{+B}}\right]^{-1}, \\
v_{-} & =\left(1-\left\langle\frac{\omega_{+}}{\omega_{-}}\right\rangle\right) /\left\langle\frac{1}{\omega_{-}}\right\rangle \\
& =\left\{1-\left[(1-c) \frac{\omega_{+A}}{\omega_{-A}}+c \frac{\omega_{+B}}{\omega_{-B}}\right]\right\}\left[\frac{1-c}{\omega_{+A}}+\frac{c}{\omega_{+B}}\right]^{-1} .
\end{aligned}
$$


Here $\omega_{+}\left(=\omega_{+A}\right.$ or $\left.\omega_{+B}\right)$ refers to the solidification frequency and $\omega_{-}\left(=\omega_{-A}\right.$ or $\left.\omega_{-B}\right)$ to the melting frequency. The angular brackets denote the ensemble average of the quantity within the brackets.

The fact that at $C_{s}$ and at $C_{l}$ the averages $S_{+}$ $=\left\langle\omega_{-} / \omega_{+}\right\rangle$and $S_{-}=\left\langle\omega_{+} / \omega_{-}\right\rangle$are equal to unity and that at these concentrations the velocities $v_{+}$and $v_{-}$vanish, respectively, reveals that the phase boundaries $C_{s}$ and $C_{l}$ represent the critical concentrations where the system undergoes a phase transition between the pinning and depinning states. In the two-phase coexistence region $C_{s}<c<C_{l}$, the mean displacement is no longer linear in time. For example, the position $h(t)$ of the solidification front instead advances as a power law $\langle h(t)\rangle \sim t^{\nu_{+}}$in the concentration range $C_{s}<c$ $<C_{0}$ with

$$
C_{0}(T)=\frac{\ln \left(\omega_{+A} / \omega_{-A}\right)}{\ln \left(\omega_{+A} \omega_{-B} / \omega_{-A} \omega_{+B}\right)} .
$$

Here the exponent $\nu_{+}$is less than unity and decreases on leaving the phase boundary $C_{s}$ : The steady-state velocity vanishes in the two-phase region. According to Derrida's $[7,8]$ general treatment of the $1 \mathrm{D}$ hopping model, the exponent $\nu_{+}$is determined from the relation

$$
\left\langle\left(\frac{\omega_{-}}{\omega_{+}}\right)^{\nu_{+}}\right\rangle=1 .
$$

In the present case, this equation can be written explicitly as

$$
(1-c)\left(\frac{\omega_{-A}}{\omega_{+A}}\right)^{\nu_{+}}+c\left(\frac{\omega_{-B}}{\omega_{+B}}\right)^{\nu_{+}}=1 .
$$

At $c=C_{0}$,

$$
\left\langle\ln \left(\omega_{-} / \omega_{+}\right)\right\rangle=0
$$

and $\nu_{+}=0$. The Monte Carlo simulation [4] gives good agreement with the theory.

For a 1D system accordingly the phase boundary at a finite temperature corresponds to the pinning-depinning transition line. The natural question is whether this statement is still valid even in the $2 \mathrm{D}$ system. We address the similarity and the difference in the two systems in this paper.

\section{TWO-DIMENSIONAL MODEL}

The 2D kinetic model for a diffusionless alloy growth was also introduced by Temkin [13]. A simple square lattice is randomly occupied by $A$ and $B$ atoms and it is decomposed into two parts by a solid-liquid interface, liquid above solid, for example. The solidification or melting takes place only at this interface: Only those atoms which have both solid and liquid nearest neighbors have the possibility to transform to the other phase. In the study reported here, atoms are not allowed to change their positions. The atomic configuration is quenched. Solidification therefore proceeds via a "diffusionless" transformation. For simplicity we assume that the cohesive energies act between nearest-neighbor atoms of the same phase but they do not depend on the type of atoms:

$$
\epsilon_{A A}^{\beta}=\epsilon_{A B}^{\beta}=\epsilon_{B B}^{\beta}=\epsilon_{\beta} \quad(\beta=s, l) .
$$

Here the superscripts $s$ and $l$ refer to the solid and liquid state, respectively. Since the mixing energy

$$
\epsilon_{A B}^{\beta}-\frac{\epsilon_{A A}^{\beta}+\epsilon_{B B}^{\beta}}{2}
$$

is zero, both phases are ideal solutions. The interface thus corresponds to the place where the atomic cohesion is broken and each broken bond depletes interface energy. We study the effect of the interface energy on the dynamics of the alloy solidification.

The phase transformation is assumed to take place stochastically at the interface. The phase change of an atom is associated with the breaking of cohesion of nearest neighboring atoms in the same phase that depletes energy. Depending on the process (freezing or melting) and on the number $j$ $(=1,2,3)$ of nearest-neighboring atoms in the same phase, there are 12 transition frequencies: six solidification frequencies denoted as $\omega_{+X}(j)$ and six melting frequencies denoted by $\omega_{-X}(j)$. Here $X$ refers to the $A$ or $B$ atom. These frequencies should satisfy the detailed balance condition to ensure the equilibrium phase diagram as

$$
\begin{aligned}
& \omega_{+X}(j)=\omega_{+X} \exp \left[-(j-2) \epsilon_{l} / T\right], \\
& \omega_{-X}(j)=\omega_{-X} \exp \left[-(j-2) \epsilon_{s} / T\right] .
\end{aligned}
$$

The transition frequencies $\omega_{ \pm X}$ of component $X$ at the kink position $(j=2)$ are related to the chemical potential difference $\Delta \mu_{X}$ between two phases as

$$
\frac{\omega_{+X}}{\omega_{-X}}=\exp \left(\frac{\Delta \mu_{X}}{T}\right)
$$

with

$$
\Delta \mu_{X}=\mu_{X}^{l}-\mu_{X}^{s} \cong L_{X} \frac{T_{X}-T}{T_{X}} .
$$

The solidus $C_{s}(T)$ and the liquidus lines $C_{l}(T)$ obey the detailed balance principle in the form

$$
\begin{aligned}
\left(1-C_{s}\right) \omega_{-A} & =\left(1-C_{l}\right) \omega_{+A}, \\
C_{s} \omega_{-B} & =C_{l} \omega_{+B},
\end{aligned}
$$

and they are obtained as in Eq. (1).

There exists a special concentration $C_{0}$ where the free energy $\phi_{s}$ per solid atom is equal to the free energy $\phi_{l}$ per liquid atom,

$$
\begin{array}{r}
\phi_{s} \equiv\left(1-C_{0}\right) \mu_{A}^{s}(T)+C_{0} \mu_{B}^{s}(T) \\
=\phi_{l} \equiv\left(1-C_{0}\right) \mu_{A}^{l}(T)+C_{0} \mu_{B}^{l}(T)
\end{array}
$$

or

$$
\left(1-C_{0}\right) \Delta \mu_{A}+C_{0} \Delta \mu_{B}=0 .
$$

From Eqs. (11) and (15), we obtain $C_{0}$ as in Eq. (4). Equation (15) is equivalent to Eq. (7) and we shall see later that the interface velocity of a large $2 \mathrm{D}$ system vanishes at this concentration $C_{0}$. 
The phase diagram with coexistence lines $C_{s}(T)$ and $C_{l}(T)$ as well as the special line $C_{0}(T)$ is depicted in Fig. 1 with the following set of parameter values: $T_{A}=0.9, T_{B}$ $=0.1, L_{A} / T_{A}=L_{B} / T_{B}=1$. It is seen that the solidification or melting of the system depends not only upon the cooling condition but also on the alloy composition. At a fixed temperature $T$ between $T_{A}$ and $T_{B}$, the system remains in the solid state as long as the concentration $c$ is smaller than $C_{s}(T)$. When $c$ comes to lie between $C_{s}(T)$ and $C_{l}(T)$, the solid and liquid phase coexist, but when $c$ becomes larger than $C_{l}(T)$ the system melts completely.

The above 2D growth model can be mapped onto the random-field Ising (RFI) model. The RFI model is defined by spins with two possible values $s_{i}= \pm 1$ that are placed on site $i$ of a lattice. Its Hamiltonian is given by

$$
\mathcal{H} \equiv-J \sum_{\langle i, j\rangle} s_{i} s_{j}-\sum_{i}\left(f_{i}+\bar{f}\right) s_{i}
$$

Here $s_{i}=+1$ represents the solid state, $s_{i}=-1$ represents the liquid state, $\Sigma_{\langle i, j\rangle}$ denotes the summation over all nearest-neighbor pairs $\langle i, j\rangle$, and $\Sigma_{i}$ is the sum over all spins in the system. $J$ is the exchange energy between the ferromagnetically coupled nearest-neighbor spins and determined by the bond energies as

$$
J=\frac{1}{4}\left(\epsilon_{s}+\epsilon_{l}\right)
$$

$\bar{f}$ is the external uniform driving force which is proportional to the concentration difference $\left(C_{0}-c\right) . f_{i}$ is the quenched local field caused by the concentration fluctuation from the average concentration $c$ of the $B$ atoms. Since each lattice site $i$ is occupied by either an $A$ atom with the probability $(1-c)$ (the average concentration of $A$ atoms) or a $B$ atom with the probability $c$ (the average concentration of $B$ atoms), the probability distribution of the quantity $\left(f_{i}+\bar{f}\right)$ is

$$
\rho\left(f_{i}+\bar{f}\right)=(1-c) \delta\left(f_{i}+\bar{f}-f_{A}\right)+c \delta\left(f_{i}+\bar{f}-f_{B}\right) .
$$

Here $f_{A}$ and $f_{B}$ depend on the temperature and the latent heat and are defined as

$$
\begin{aligned}
& f_{A}(T)=-\frac{1}{2} \Delta \mu_{A}=-\frac{1}{2} L_{A}\left(1-\frac{T}{T_{A}}\right), \\
& f_{B}(T)=-\frac{1}{2} \Delta \mu_{B}=-\frac{1}{2} L_{B}\left(1-\frac{T}{T_{B}}\right) .
\end{aligned}
$$

In addition, the driving force $\bar{f}(T)$ or the average free energy gain by solidifying one atom is

$$
\bar{f}(T)=(1-c) f_{A}(T)+c f_{B}(T),
$$

where $c$ is the average concentration of $B$ atoms. Accordingly, the randomly quenched field $f_{i}$ has a zero average value

$$
\left\langle f_{i}\right\rangle=0
$$

Here the angular brackets represent the average over the different realization of randomness. $f_{i}$ is then assumed uncor- related for different sites. It may be worthwhile to note that the two-dimensional case corresponds to the lower critical dimension of the RFI model [19].

\section{DIFFUSIONLESS GROWTH AT LARGE BOND ENERGY}

In this section we show analytically that for large bond energies a two-dimensional growth system with finite width $N$ will be equivalent to a one-dimensional system. This type of behavior is well known to occur in critical phenomena of equilibrium statistical mechanics. We consider the alloy solidification starting from a flat interface configuration. The solidification is initiated by the formation of a nucleus consisting of a single atom with two kinks sitting on a flat onedimensional interface. If the kink formation energy $\epsilon_{l}$ is large, this nucleation process is very slow compared to the lateral growth of the island afterwards. The solidification proceeds layer-by-layer by the single nucleation and growth mechanism and the probability of multilayers and multinuclei processes is negligibly small: Once a cluster appears, it soon spreads laterally until the whole layer is filled. If the bond energy $\epsilon_{s}$ is large, the melting also proceeds by a layerby-layer mechanism. In these cases the advancement of a flat interface by one layer to the neighboring layer position may be considered as a single "elementary" action. Then one may construct an effective 1D model where the "elementary" transition frequencies $\Omega_{ \pm}$of a "layer" jumping forward $(+)$ and backward $(-)$ play the same role as $\omega_{ \pm}$play in a real $1 \mathrm{D}$ system. In reality these "elementary", jumps consist of many atomic solidification-melting subprocesses. We can calculate the elementary frequencies $\Omega_{ \pm}$in terms of atomic frequencies $\omega_{ \pm}$. From the analysis, we shall find the remarkable result that the exponent $\nu$ obeys the same relation (5) as in the 1D system. This result will be tested by the Monte Carlo simulation in the next section.

First consider the situation when the layer has just been completed and the flat interface is located between the solid layer with an atomic arrangement $\left\{X^{s}\right\}=\left\{X_{1}^{s}, X_{2}^{s}, \ldots, X_{N}^{s}\right\}$ and the liquid layer above with an arrangement $\left\{X^{l}\right\}$ $=\left\{X_{1}^{l}, X_{2}^{l}, \ldots, X_{N}^{l}\right\}$. Here $X^{s, l}$ denote $A$ or $B$ atoms and $i$ $(=1,2, \ldots, N)$ denotes the column number in the square lattice. The solidification, for instance, starts by the nucleation at a site $i$ in the liquid layer. The solid nucleus grows to the right or to the left, but can also melt back. Finally the layer is completed by the solidification at the last site $f$. Here we call the sequence of events starting from the site $i$ and ending at the site $f$ a "trajectory." After an average waiting time $Y$, the interface has moved one layer forward with a probability $P$ or one layer backward with a probability $Q$, where $P+Q=1$ obviously. Then the transition frequencies of layer jump are given by

$$
\Omega_{+}=P / \Upsilon \quad \text { and } \Omega_{-}=Q / \Upsilon
$$

The forward transition probability $P$ obeys the following recursive equation, with definitions given afterwards:

$$
P=\sum_{i=1}^{N} p_{i} P_{i}+P \sum_{i=1}^{N}\left[p_{i}\left(1-P_{i}\right)+q_{i}\left(1-Q_{i}\right)\right] .
$$

Here 


$$
p_{i}=\frac{\omega_{+i}(3)}{\sum_{i=1}^{N} \omega_{+i}(3)+\sum_{j=1}^{N} \omega_{-j}(3)} \equiv \omega_{+i}(3) \tau
$$

is the probability that a solid island first nucleates at the site $i$ on the initial flat interface. Similarly

$$
q_{i}=\omega_{-i}(3) \tau
$$

gives the probability of the first melting of a solid layer at the $i$ th site. We introduced the abbreviated notation $\omega_{ \pm i}$ for $\omega_{ \pm X_{i}^{l}} . \tau$ represents the mean waiting time before the first solid or liquid nucleus is created on the flat interface.

In Eq. (23), $P_{i}$ represents the probability that the solid cluster which started from the site $i$ completely covers the interface without being remelted before finishing the coverage, so that the interface never comes back to the initial flat configuration during the solidification process. Similarly $Q_{i}$ defines the probability that the liquid cluster started from the site $i$ completely melts the interface layer without being completely resolidified to the original interface configuration.

Therefore the first term on the right-hand side (RHS) of Eq. (23) represents the contribution from all the possible trajectories which never come back to the initial flat interface during the solidification process. The second term gives the contribution from those trajectories which lead to the initial interface configuration during solidification. Those started by solidification but remelted have a probability $p_{i}\left(1-P_{i}\right)$ to return to the original interface configuration, and those started by melting and resolidified have a probability $q_{i}\left(1-Q_{i}\right)$. Afterwards the interface has the same probability $P$ to be solidified. Equation (23) has a solution

$$
P=\frac{\sum_{i=1}^{N} p_{i} P_{i}}{\sum_{i=1}^{N}\left[p_{i} P_{i}+q_{i} Q_{i}\right]} .
$$

Obviously $Q=1-P$. In the same spirit we can derive the equation for the average waiting time $Y$ :

$$
\begin{aligned}
\Upsilon= & \tau \sum_{i=1}^{N}\left[p_{i} P_{i}+q_{i} Q_{i}\right]+(\tau+\Upsilon) \\
& \times \sum_{i=1}^{N}\left[p_{i}\left(1-P_{i}\right)+q_{i}\left(1-Q_{i}\right)\right] .
\end{aligned}
$$

Equation (27) is approximate because we take into account only a single nucleation and growth during the interface evolution.

It has the solution

$$
\Upsilon=\frac{\tau}{\sum_{i=1}^{N}\left[p_{i} P_{i}+q_{i} Q_{i}\right]}
$$

Now we obtain the desired transition frequencies:

$$
\begin{gathered}
\Omega_{+}=\frac{1}{\tau} \sum_{i=1}^{N} p_{i} P_{i}=\sum_{i=1}^{N} \omega_{+i}(3) P_{i}, \\
\Omega_{-}=\sum_{i=1}^{N} \omega_{-i}(3) Q_{i} .
\end{gathered}
$$

The important feature of the obtained result, Eq. (29), is that in the used approximation the frequency of solidification, $\Omega_{+}$, depends only on the atomic configuration of the solidifying liquid layer above the interface. Similarly the melting frequency $\Omega_{-}$depends only on the atomic configuration of the melting solid layer at the interface.

In terms of the introduced frequencies $\Omega_{ \pm}$, we can write down some important characteristics using the known 1D results. The solidification velocity $v_{+}$and the melting velocity $v_{-}$are given as

$$
\begin{aligned}
& v_{+}=+\left[1-\left\langle\frac{\Omega_{-}}{\Omega_{+}}\right\rangle\right] /\left\langle\frac{1}{\Omega_{+}}\right\rangle, \\
& v_{-}=-\left[1-\left\langle\frac{\Omega_{+}}{\Omega_{-}}\right\rangle\right] /\left\langle\frac{1}{\Omega_{-}}\right\rangle .
\end{aligned}
$$

Here $\langle\cdots\rangle$ means the average over all possible configurations in the layer, for example,

$$
\left\langle\frac{\Omega_{-}}{\Omega_{+}}\right\rangle=\sum_{X_{1}=A, B} \cdots \sum_{X_{N}=A, B} \frac{\Omega_{-}\left(X_{1} \cdots X_{N}\right)}{\Omega_{+}\left(X_{1} \cdots X_{N}\right)} \prod_{i=1}^{N} c_{i},
$$

where $\Pi_{i=1}^{N} c_{i}$ (with $c_{i}=c$ for $X_{i}=B$ and $c_{i}=1-c$ for $X_{i}$ $=A)$ is the probability of finding the configuration $\left\{X_{i} \cdots X_{N}\right\}$. These steady-state velocities, $v_{+}$and $v_{-}$, vanish under the following conditions:

$$
\left\langle\Omega_{-} / \Omega_{+}\right\rangle=1 \quad \text { and }\left\langle\Omega_{+} / \Omega_{-}\right\rangle=1,
$$

respectively. In the region where steady-state motion of the interface is impossible, the mean displacement $\langle h(t)\rangle$ shows a power-law behavior $\left\langle h_{+}(t)\right\rangle \sim t^{\nu_{+}}$and $\left\langle h_{-}(t)\right\rangle \sim-t^{\nu_{-}}$ with the exponents $\nu_{+}$and $\nu_{-}$determined from the relations

$$
\left\langle\left(\Omega_{-} / \Omega_{+}\right)^{\nu_{+}}\right\rangle=1 \quad \text { and }\left\langle\left(\Omega_{+} / \Omega_{-}\right)^{\nu_{-}}\right\rangle=1 \text {. }
$$

At the point where

$$
\left\langle\ln \left(\Omega_{-} / \Omega_{+}\right)\right\rangle=0,
$$

the mean displacement vanishes, $\left\langle h_{+}(t)\right\rangle=\left\langle h_{-}(t)\right\rangle=0$, and $\nu_{+}=\nu_{-}=0$. Note that in accord with the $1 \mathrm{D}$ results $[1-3]$, the frequencies $\Omega_{-}$and $\Omega_{+}$in Eqs. (30)-(34) should be taken for the same layer.

In order to evaluate the elementary layer-jump frequencies of Eq. (22), we need the probabilities $P_{i}$ and $Q_{i}$ in Eq. (29). For $P_{i}$ with $i=1,2, \ldots, N$, one can write the following set of equations: 


$$
\begin{aligned}
& P_{i}=\frac{1}{W_{i, i}}\left\{\omega_{+(i-1)} P_{i-1, i}+\omega_{+(i+1)} P_{i, i+1}\right\}, \\
& P_{i, \ldots, i+n}= \frac{1}{W_{i, i+n}}\left\{\omega_{-i} P_{i+1, \ldots, i+n}\right. \\
&+\omega_{-(i+n)} P_{i, \ldots, i+n-1}+\omega_{+(i-1)} \\
& \times P_{i-1, \ldots, i+n}+\omega_{+(i+n+1)} \\
&\left.\times P_{i, \ldots, i+n+1}\right\}, \\
& P_{i, \ldots, i+N-2} \frac{1}{W_{i, i+N-2}}\left\{\omega_{-i} P_{i+1, \ldots, i+N-2}\right. \\
&+\omega_{-(i+N-2)} P_{i, \ldots, i+N-3} \\
&\left.+\omega_{+(i+N-1)}(1)\right\} .
\end{aligned}
$$

The $Q_{i}$ 's obey completely analogous equations apart from index changes, but an explicit evaluation can be circumvented as shown in the Appendix. Here now $n=1, \ldots, N$ -3 and $P_{i, \ldots, i+n}$ is the probability that the solid cluster with $(n+1)$ particles which occupies the sites from $i$ to $(i+n)$ finally fills the layer of $N$ atoms without being completely remelted. The denominators are the total rate of possible events for the nucleus occupying the sites $i$ to $i+n$,

$$
\begin{gathered}
W_{i, i}=\omega_{-i}(1)+\omega_{+(i-1)}+\omega_{+(i+1)}, \\
W_{i, i+n}=\omega_{-i}+\omega_{-(i+n)}+\omega_{+(i-1)}+\omega_{+(i+n+1)}, \\
W_{i, i+N-2}=\omega_{-i}+\omega_{-(i+N-2)}+\omega_{+(i+N-1)}(1) .
\end{gathered}
$$

We consider Eqs. (35) and (36) with periodic boundary conditions. Namely, $\omega_{ \pm(k+N)}=\omega_{ \pm k}$, and a cluster which reaches a boundary of the layer can continue to grow from the other side of the layer.

For a given configuration of $A-B$ atoms in the layer, one can solve the linear equations (35) numerically. A closed analytical form of the solution is obtained for $N=2,3,4$ and is summarized in the Appendix. The remarkable result found in the analysis is the relation

$$
\frac{\Omega_{-}}{\Omega_{+}}=\prod_{i=1}^{N}\left(\frac{\omega_{-i}}{\omega_{+i}}\right)
$$

for $N=2,3,4$. So far this result has been explicitly shown only for systems with finite sizes $N=2,3,4$, but it can be extended to an arbitrarily large $N$ by a plausible argument. It is clear that each trajectory which gives a contribution to $\Omega_{+}$ has the common factor $\Pi_{i=1}^{N} \omega_{+i}$, because all atoms in the liquid layer have to be solidified at least once. An exponential factor contained at the initiation of a solid nucleus, $\omega_{+1}(3)=\omega_{+1} \exp \left(-\epsilon_{l} / T\right)$, is compensated for by the factor at the end: $\omega_{+N}(1)=\omega_{+N} \exp \left(\epsilon_{l} / T\right)$. Index " 1 " here simply is the place where the nucleation of the new layer has started. Additional melting and resolidification at the site $j$ in the intermediate stage introduces a factor $\omega_{-j} \omega_{+j}$. Thus we can formally write $\Omega_{+}$as a sum over all possible trajectories,

$$
\Omega_{+}=\left(\prod_{i=1}^{N} \omega_{+i}\right) \sum_{k} f_{+k}\left(\left\{\omega_{-j} \omega_{+j}\right\}, W_{n, m}\right),
$$

where the function $f_{+k}$ describes the contribution from the $+k$ th trajectory. $W_{n, m}$ is given in Eq. (36). A similar formal expression can be written for $\Omega_{-}$for the same layer:

$$
\Omega_{-}=\left(\prod_{i=1}^{N} \omega_{-i}\right) \sum_{k} f_{-k}\left(\left\{\omega_{+j} \omega_{-j}\right\}, W_{n, m}\right) .
$$

We cannot calculate the trajectory sum in the general case, but we can show that $\Sigma_{k} f_{+k}=\Sigma_{k} f_{-k}$. For each $+k$ th trajectory there exists a $-k$ th trajectory with precisely the opposite sequence of elementary events, like a film running forward for the $+k$ th trajectory and backward for the $-k$ th one. Through this one-to-one correspondence between trajectories, one gets $f_{+k}=f_{-k}$ and the relation Eq. (37).

From the property, Eq. (37), that the ratio $\Omega_{-} / \Omega_{+}$is just the product of the ratios of the frequencies $\omega_{-i} / \omega_{+i}$, we can now derive that the 2D system with large $\epsilon_{s}$ behaves similarly to the pure $1 \mathrm{D}$ system. The average of the ratio reduces to

$$
\left\langle\frac{\Omega_{-}}{\Omega_{+}}\right\rangle=S_{+}^{N}
$$

with

$$
S_{+}=\left\langle\frac{\omega_{-i}}{\omega_{+i}}\right\rangle=(1-c) \frac{\omega_{-A}}{\omega_{+A}}+c \frac{\omega_{-B}}{\omega_{+B}} .
$$

From Eq. (30) the growth rate $v_{+}$then vanishes at $S_{+}=1$. This is precisely the same condition for the absence of steady growth in the 1D system Eq. (3), and it corresponds to the solidus line on the phase diagram. Moreover, Eq. (33) for the exponent $\nu_{+}$has the form

$$
\left[(1-c)\left(\frac{\omega_{-A}}{\omega_{+A}}\right)^{\nu_{+}}+c\left(\frac{\omega_{-B}}{\omega_{+B}}\right)^{\nu_{+}}\right]^{N}=1
$$

and gives the same exponent $\nu_{+}$as for the 1D system [see Eq. (6)]. All these results obtained for the solidification front can be easily converted into the melting front just by exchanging the indices + and - . For example, the melting velocity $v_{-}$vanishes at the concentration

$$
S_{-} \equiv(1-c) \frac{\omega_{+A}}{\omega_{-A}}+c \frac{\omega_{+B}}{\omega_{-B}}=1
$$

\section{NUMERICAL RESULTS AND DISCUSSIONS}

\section{A. Implementation of the algorithm}

For the analysis of the 2D alloy solidification with general values of bond energies and arbitrary system size $N$, we perform Monte Carlo (MC) simulations of a lattice model. On a square lattice with $N$ columns and $M$ rows, $A$ and $B$ atoms are distributed randomly with a mean concentration $c$ of the $B$ component. Each atom can be in either a solid or a liquid state. We use the solid-on-solid (SOS) model such that the liquid phase stays above the solid phase: Overhangs are forbidden at the interface. Then, an interface configuration is 
defined as the set of heights of the solid phase $\left\{h_{i}\right\}$ for columns $i=1$ to $N$. Initially an interface is flat at a height $h_{i}(t=0)=h(0)$ for all the columns. Each row is assumed periodic and both ends of each column are assumed free.

For the motion of the interface, only $2 \mathrm{~N}$ atoms contingent to the interface are involved. Let us denote the interface configuration as $\left\{h_{i}\right\}$, the solid-atom arrangement at the interface as $\left\{X^{s}\left(h_{i}\right)\right\}$, and the liquid-atom arrangement as $\left\{X^{l}\left(h_{i}+1\right)\right\}$. The melting rate of a solid atom on the $i$ th column depends on the number $j_{i}$ of neighboring solid atoms as $\omega_{-i} \equiv \omega_{-X^{s}\left(h_{i}\right)}\left(j_{i}\right)$ and the solidification rate of a liquid atom above the interface depends on the number $j_{i}^{\prime}$ of neighboring liquid atoms as $\omega_{+i} \equiv \omega_{+X^{l}\left(h_{i}+1\right)}\left(j_{i}^{\prime}\right)$. Then, during the time interval

$$
\Delta t=1 /\left(\sum_{i=1}^{N} \omega_{+i}+\sum_{i=1}^{N} \omega_{-i}\right),
$$

an atom in the $i$ th column in the liquid or solid layer changes its state with the transition probability

$$
P_{ \pm}(i)=\omega_{ \pm i} \Delta t
$$

where $i=1, \ldots, N$. Here $+(-)$ refers to the solidification (melting) of the atom at the height $h_{i}+1\left(h_{i}\right)$. Since the probability (45) is normalized, some event takes place within the time interval $\Delta t$. Therefore, our simulation algorithm runs as follows. Pick up a random number between 0 and 1 . If it falls in the interval between $\sum_{n=1}^{i-1} P_{+}(n)$ and $\sum_{n=1}^{i} P_{+}(n)$, then the liquid atom at the site $\left(i, h_{i}+1\right)$ solidifies and the interface height increases. If the random number falls in the interval between $\sum_{n=1}^{N} P_{+}(n)$ $+\sum_{n=1}^{i-1} P_{-}(n)$ and $\sum_{n=1}^{N} P_{+}(n)+\sum_{n=1}^{i} P_{-}(n)$, then the solid atom at the site $\left(i, h_{i}\right)$ melts and the interface height decreases. After each configuration change the time increases by $\Delta t$. The mean displacement of the interface at the time $t$ is defined as

$$
\langle h(t)\rangle=\frac{1}{N} \sum_{i=1}^{N}\left\langle h_{i}(t)\right\rangle-h(0),
$$

where $\langle\cdots\rangle$ represents the sample average and $h(0)$ is the initial position of the interface. Averages of physical quantities have been taken from 50 to 150 independent samples.

With the present algorithm, we reproduced the results of a 1D diffusionless solidification obtained previously [4]. In addition, we simulated a two-column system which is composed of one column of pure $A$ atoms and one column of pure $B$ atoms. For this system we can calculate the velocity of the interface exactly and the simulation reproduces the exact velocity quite well. For a further check of our program, the equilibrium distribution of kink height is investigated for a 2D single-component system and the Boltzmann distribution is confirmed.

\section{B. Large $\epsilon$ limit}

In Sec. IV we proved that when the bond energies $\epsilon_{s}$ are infinitely large and the system width is finite, the 2D system has asymptotics equivalent to the $1 \mathrm{D}$ system. In order to check this, we simulate the interface motion of a four-

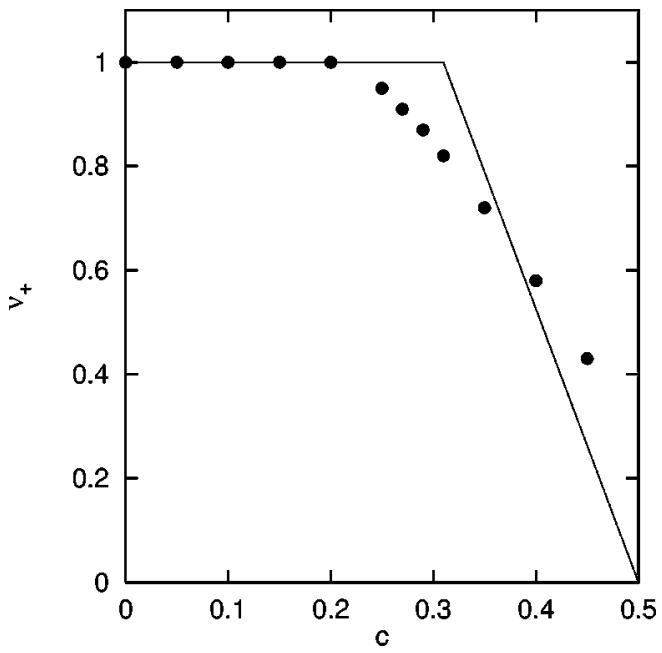

FIG. 2. Exponent $\nu_{+}$of the mean displacement at various concentrations of $B$ atoms for the four-column system: $N=4$. The solid line represents the theoretical expectation Eq. (47) and the dots represent the $\mathrm{MC}$ results.

column lattice: $N=4$. The parameters governing the phase diagram are $T_{A}=0.9, T_{B}=0.1$, and $L_{A} / T_{A}=L_{B} / T_{B}=1$. The correspondent phase diagram is shown in Fig. 1. Other parameters are chosen as $\epsilon_{s} / T_{A}=\epsilon_{l} / T_{A}=3.0$ and $T=0.5$. Frequencies are chosen as $\omega_{-A}=\omega_{-B}=1$, which defines our unit time, and $\omega_{+A}=1 / \omega_{+B}=2.226$. The equilibrium concentrations at this temperature are $C_{s}=0.310$ and $C_{l}$ $=0.690$. By solving Eqs. (11), (12), and (6), the exponent for the displacement of the interface is obtained as

$$
\nu_{+}=1.25 \ln \left(\frac{1-c}{c}\right) \quad(0.31<c<0.50) .
$$

Since the bond energies $\epsilon_{s} / T=\epsilon_{l} / T=5.4$ are large, the thermally excited kink density is small; $\exp \left(-\epsilon_{l} / T\right)$ is of the order of $10^{-3}$. Then the solid-liquid interface is expected to advance by the single nucleation and growth mechanism as is described in Sec. IV.

The exponent $\nu_{+}$and the velocity $v_{+}$obtained by the simulation are presented as functions of the concentration $c$ in Fig. 2 and Fig. 3, respectively. The velocity in Fig. 3 is determined from the asymptotic slope of the displacement versus time and it has a nonzero value even in the two-phase region. The solid line in Fig. 2 represents the theoretical behavior Eq. (47). The solid line in Fig. 3 is the velocity Eq. (A7) obtained theoretically for $N=4$. The deviation of the exponent $\nu_{+}$obtained by simulation from the theoretical value near the phase boundary $C_{s}$ is also observed in a $1 \mathrm{D}$ case [4] and is probably due to the finite simulation time. Comparing the analytic prediction (solid line) with the simulation results (symbols), we can conclude that the 2D diffusionless crystal growth in the infinite bond energy limit obeys the same law as the $1 \mathrm{D}$ counterpart. It means that the interface advances steadily in the one-phase region, but the steady-state velocity vanishes in the two-phase region.

\section{The effect of bond energy $\epsilon$ and system size $N$ on the interface properties}

It has been known [13] that the interface of a 2D alloy system has a steady-state solidification velocity in the solid 


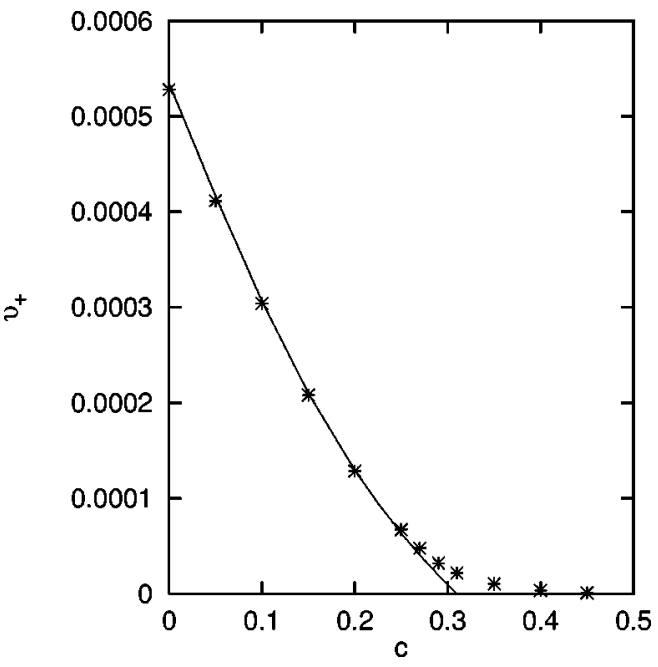

FIG. 3. Steady-state velocity $v_{+}$versus concentration $c$ for the four-column system: $N=4$. The solid line represents the theoretical expectation Eq. (A7), and the dots represent the MC results.

phase region $\left(c<C_{s}\right)$ and a steady-state melting velocity in the liquid phase region $\left(c>C_{l}\right)$. Naturally, the basic question we want to ask is how the interface in the two-phase coexistence region behaves. There are many parameters in the system, but the ones in the $2 \mathrm{D}$ system which are new compared to the 1D system are the bond energy $\epsilon$ and the system size $N$. Therefore, we study the effect of these two parameters on the dynamics of the interface. The other parameter values are kept fixed to reproduce the same equilibrium phase diagram, Fig. 1: $T_{A}=0.9, T_{B}=0.1, L_{A} / T_{A}$ $=L_{B} / T_{B}=1$. We choose the concentration $c=0.4$ and the temperature $T=0.5$ such that the corresponding phase point is located in the two-phase coexistence region with a solid phase favored (see Fig. 1). Then the choice of $\omega_{-A}=\omega_{-B}$ $=1$ leads to solidification frequencies $\omega_{+A}=1 / \omega_{+B}=2.226$ at $T=0.5$. For simplicity, the bond energies $\epsilon_{s}$ and $\epsilon_{l}$ take always the same value: $\epsilon_{s}=\epsilon_{l}=\epsilon$.

We first study the time dependence of the interface displacement $\langle h(t)\rangle$ for various system sizes $N$ at a given bond

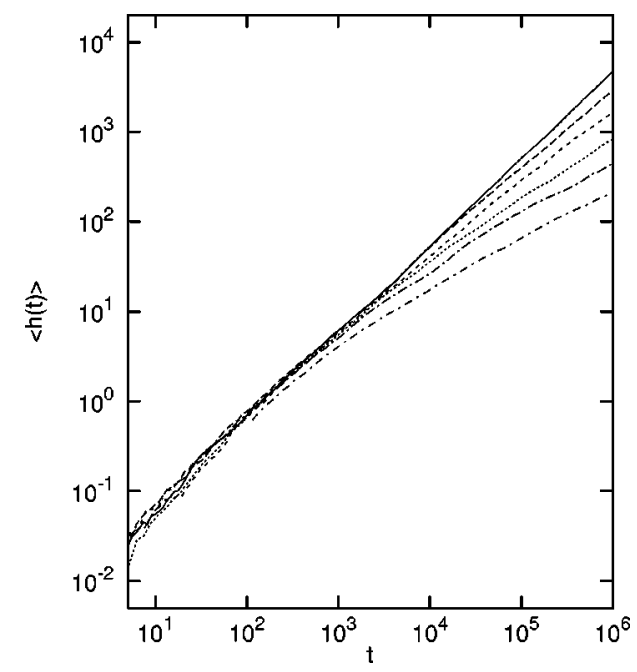

FIG. 4. Log-log plot of the mean displacement $\langle h(t)\rangle$ as a function of time $t$ for various system sizes: $N=20,30,40,50,60$, and 80 from the bottom to the top. The bond energy is $\epsilon=2.0 T_{A}$.

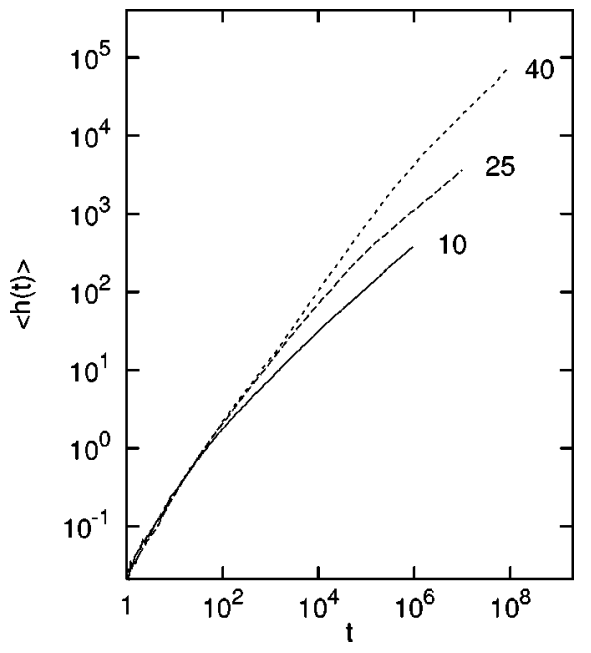

FIG. 5. Log-log plot of the mean displacement $\langle h(t)\rangle$ as a function of time $t$ for three system sizes: $N=10,25$, and 40 . The bond energy is $\epsilon=1.5 T_{A}$.

energy $\epsilon$. Averages have been taken over 50 to 150 independent runs. Figure 4 depicts the raw data for six different sizes $N$ ranging from 20 to 80 at the fixed bond energy $\epsilon / T_{A}$ $=2.0$. The simulation is performed up to the time $10^{6}$. Initially the displacement $h(t)$ increases linearly with time $t$ independent of the system size $N$. For a time longer than the crossover time $t_{x}(N)$ which depends on the system size, the system crosses over to a pinned state with the displacement following a power law as $h(t)=a t^{\nu}+b$. Up to a simulation time $t=10^{6}$ we obtain a dynamic exponent $\nu$ which varies from around 0.51 to 1 as the system size $N$ increases from 25 to 80 . The same tendency is also observable for a system with a smaller bond energy, $\epsilon / T_{A}=1.5$, as is depicted in Fig. 5. Here the simulation is performed longer up to the time $t$ $=10^{8}$. The displacement $\langle h(t)\rangle$ clearly shows the crossover from the steadily moving interface to the pinned growth.

In the simulation, the solidification starts from a straight interface. As atoms on the interface change their state between the liquid and the solid states, the interface gradually roughens and bumps appear. At the beginning, bumps are small and isolated from each other. Therefore the interface does not feel the size differences among different systems and displays a common linear law. However, at a crossover time when the typical bump size reaches the system size, the system is affected strongly by its size. In the long time limit all systems lose the memory of the size and present a quasi-1D behavior, which is characterized by the power-law behavior of the displacement. This scenario will be further elucidated later in Sec. V G.

Next we examine the effect of the bond energy $\epsilon$ on the dynamic exponent $\nu$ for a given system size $N$. The (effective) exponent $\nu$ is obtained from the simulation up to the time $t=10^{5}-10^{6}$. We studied seven systems with sizes ranging from 25 to 2000 and the result is summarized in Fig. 6. It clearly shows that there is an evident size and bond energy dependence of the interface dynamics. We can divide the bond energy dependence of $\nu$ into four characteristic regimes (a), (b), (c), and (d) - as is exemplified in Fig. 6 for the system with a size $N=40$. 


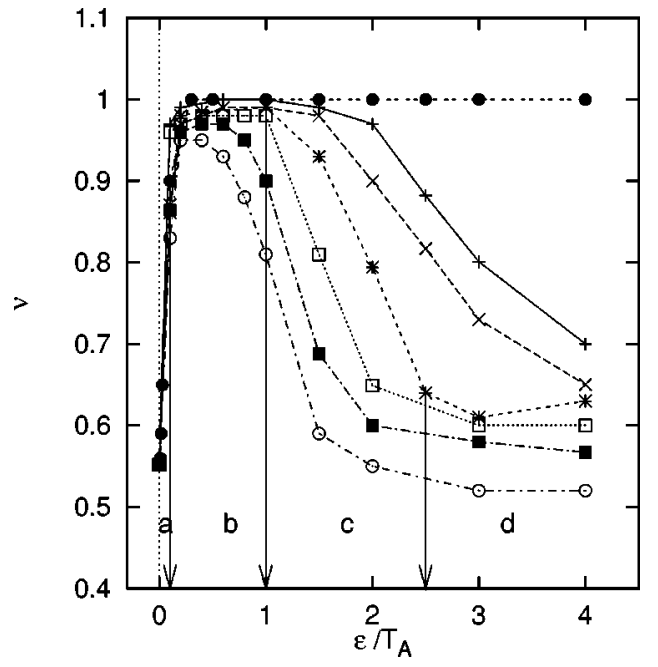

FIG. 6. Dependence of the dynamical exponent $\nu$ on the bond energy $\epsilon$. Curves from bottom to top correspond to system sizes $N=25,30,40,50,70,80$, and 2000. Data are obtained from the simulations performed up to the time $10^{6}$.

(a) Jump regime. The exponent $\nu$ increases sharply from the $1 \mathrm{D}$ value, 0.51 , to unity in a very short range of energy from 0 to $0.1 T_{A}$.

(b) Steady-state velocity regime. For moderate bond energies $\left(>0.1 T_{A}\right)$, the interface moves steadily and its displacement grows linearly with time; the exponent $\nu$ is 1 . This is quite a surprise since the interface of a $1 \mathrm{D}$ chain gets completely pinned at the concentration $c=0.4$. The energy range where $\nu$ remains unity varies with the system size. The larger the system size $N$, the wider the energy range becomes.

(c) Transit regime. If the bond strength $\epsilon$ exceeds the critical value $\epsilon_{c}(N)$, then $\nu$ starts to decrease. We define $\epsilon_{c}$ as the value of the bond energy when $\nu$ drops to 0.9. The critical value $\epsilon_{c}$ is found to increase linearly with the system size $N$, as is shown in Fig. 7.

(d) Pinned regime. For a strong enough bond energy $\epsilon$, the exponent $\nu$ eventually approaches the $1 \mathrm{D}$ value 0.51 .

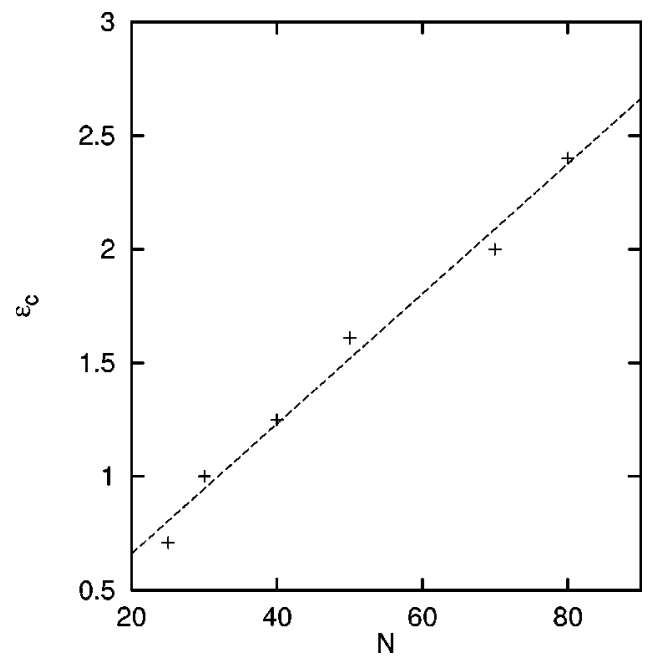

FIG. 7. Dependence of critical bond energy $\epsilon_{c}$ on the system size $N$.

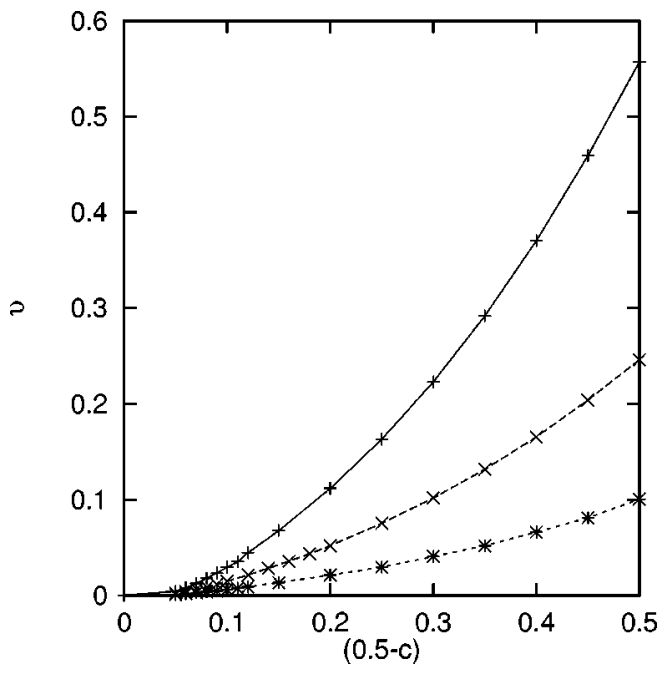

FIG. 8. Dependence of the velocity $v$ on the concentration difference $(0.5-c)$. The system size is $N=2000$ and the bond energies are $\epsilon=1.0 T_{A}(+), 1.5 T_{A}(\times)$, and $2.0 T_{A}(*)$, respectively.

This regime has been studied in detail in Sec. IV and the Appendix theoretically and in Sec. V B by the simulation.

\section{Depinning of large systems}

In Sec. VC, we have seen that the properties of the 2D system depend strongly on two parameters: the size $N$ and the bond energy $\epsilon$. In the limit of large energy $(\epsilon \rightarrow \infty)$, the system loses its 2D features and behaves effectively like its 1D counterpart. On the contrary, in the limit of large system size $(N \rightarrow \infty)$, the crystal grows steadily for the concentrations $c<C_{0}(T)$ if $\epsilon>0.1 T_{A}$ (see Fig. 6 for $N=2000$ ). Here the critical concentration $C_{0}$ is defined in Eq. (4) and assumes the value 0.5 at $T=0.5$. In the one-phase region, 0 $\leqslant c \leqslant C_{s}$, it is found that the steady velocity decreases as the bond energy $\epsilon$ increases (see Fig. 8). The ratio of velocities of two systems with bond energies $\epsilon_{1}$ and $\epsilon_{2}$ is found to be almost independent of the concentration when the bond energy is larger than $\approx T_{A}$, while $T_{A}$ is the high-temperature limit of the two-phase region at $c=0$ (Fig. 1). In other words, the ratio is a function of $\epsilon_{1}$ and $\epsilon_{2}$ as $v\left(\epsilon_{1}, c\right) / v\left(\epsilon_{2}, c\right)=f\left(\epsilon_{1}, \epsilon_{2}\right)$. However, for small bond energies the ratio depends also on the concentration. So far, we know of no theory on the velocity in the one-phase region in two dimensions.

Our concern hereafter in this section is the growth law in the two-phase region. How does the growth rate depend on the driving force $H \propto C_{0}-c$ for a very large system in the two-phase region? Figure 8 shows the simulation results of the velocity of the solidification front as a function of the driving force $H \propto 0.5-c$ for a large system with size $N$ $=2000$. Three different bond energies are considered: $\epsilon / T_{A}$ $=1.0,1.5$, and 2.0. The simulation has been performed only once for each parameter set, but statistics are sufficiently good due to the large system size.

Figure 8 clearly shows that the velocity of the front increases systematically with the driving force $H$. We study the interface motion under a small driving force $H$ in the spirit of a driven elastic manifold [15-18]. Our picture of the interface motion is as follows. In the absence of an external driving force $H=0$ or $c=C_{0}=0.5$, the interface moves until 


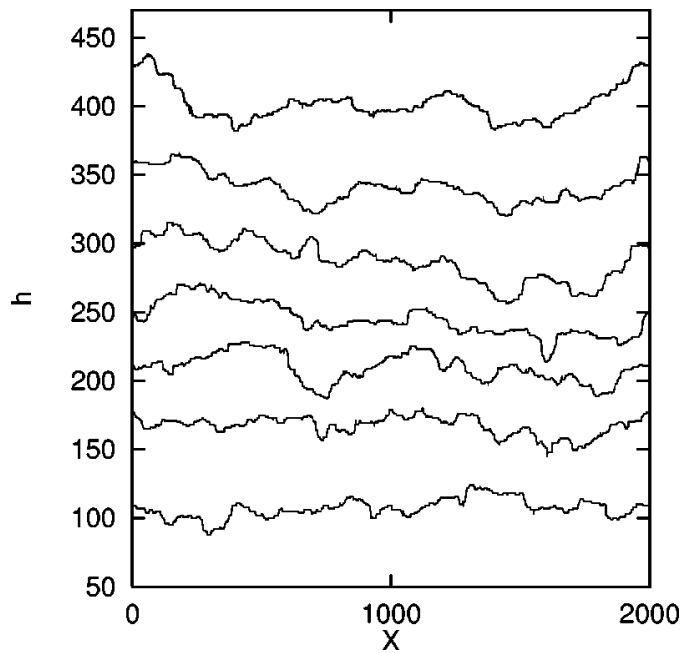

FIG. 9. Time evolution of an interface profile of a $2 \mathrm{D}$ system under a small driving force: $0.5-c=0.08$. The system size is $N$ $=2000$ and the bond energy is $\epsilon=2.0 T_{A}$. Note that the vertical and horizontal scales are different.

it finds a configuration with a local minimum of the energy, whereupon it is pinned. If we drive a system with a nonzero force

$$
H=b(0.5-c)
$$

the interface tends to move in the direction of $H$, but the pinning force of the impurities tends to block the motion. Here $b$ is a constant. As soon as the pinning force is overcome by the external force $H$ and the restoring force generated by the local curvature of the interface, the interface begins to move with a finite velocity. For a very small field $H$ the motion may not be uniform. At a given instant the interface may consist of pinned and unpinned regions. The height difference between the pinned and unpinned region increases in time, and thus the interface should be randomly corrugated with large amplitudes. Typical interface profiles are shown in Fig. 9. The eye can readily recognize that the roughness increases in time. But once the combined effect of the driving and restoring forces overcomes the pinning forces in some pinned region, the interface jumps over the pinning sites and begins to move until it is stopped again by another region of strong pinning sites. Thus the interface exhibits a slow, smooth motion stopped by pinning. After waiting times which are typically long in comparison with the time to move from one pinned state to the next, the interface jumps over the pinning center and then moves on continuously until the next stop at a pinning site. The interface velocity $v$ thus is expected to be inversely proportional to the waiting time $t_{w}$ for a jump.

We now estimate this waiting time $t_{w}$. The randomly quenched $B$ atom on the site $i$ corresponds to a pinning center against the solidification front with a pinning force $f_{i}$. Let us consider the case that the interface has to jump over a pinning region with an extension $l$ and a height $h$. This region pins the solidification front because it has more $B$ atoms than the expected average value and the liquid phase is favored with an energy gain $\sim \Delta(h l)^{1 / 2}$. Here $\Delta$ is a prefactor characterizing the effective strength of the random pinning force and is proportional to the quantity $\left\langle f_{i} f_{j}\right\rangle$. For the interface to pass over this region, it has to pay the energy penalty

$$
u_{r} \sim \Delta(h l)^{1 / 2} .
$$

However, the interface deformation depletes surface energy and therefore the jump gains the surface energy

$$
u_{s} \sim \gamma\left(\frac{h}{l}\right)^{2} l
$$

where $\gamma$ is the surface tension. If there is a bulk driving force $H$, the solidification of the volume $h l$ leads to the energy gain

$$
u_{d} \sim H h l \text {. }
$$

By assuming that the jump takes place when all the competing energies are of the same order as $u_{s} \sim u_{r} \sim u_{d}$, the pinning region to be jumped over has the following characteristic length scales:

$$
l \sim \gamma^{1 / 3} \Delta^{2 / 3} / H, \quad h \sim \Delta^{4 / 3} / H \gamma^{1 / 3},
$$

and the characteristic energy

$$
u=d \Delta^{2} / H,
$$

where $d$ is some constant of order unity. Assigning this to be the height of the energy barrier over which the interface has to jump, the waiting time for the interface is estimated as $t_{w} \sim e^{u / T}$. The velocity of the interface is simply the inverse of the waiting time, and can be expressed as

$$
v=g \exp \left(\frac{-d \Delta^{2}}{H T}\right),
$$

where $g$ is a prefactor. Thus for small driving $H$ the velocity $v$ is expected to be exponentially decreasing with $H^{-1}$. In order to check this, we take the logarithm of both sides of the formula (54) and by using the relation Eq. (48) we obtain the relation

$$
(0.5-c) \ln (v)=-\frac{d \Delta^{2}}{b T}+(0.5-c) \ln (g) .
$$

As the driving force $(0.5-c)$ approaches zero, the value of $(0.5-c) \ln (v)$ decreases linearly and reaches the value $-d \Delta^{2} / b T$ at $H=0$. The linear law is verified by the numerical simulation as is shown in Fig. 10. For three different bond energies $\epsilon / T_{A}=1,1.5$, and 2 , data points behave linearly in the vicinity of zero driving force and all linearly fitted lines cut the $y$ axis at the point -0.192 at $H=0$. Since the temperature is $T=0.5$, so $d \Delta^{2} / b$ is estimated about 0.096 .

We shall now estimate the constant $b$ and the strength of the pinning force $\Delta$. One starts from the analysis of the free energy of a binary lattice system without concentration fluctuation. By solidifying $M=h l$ atoms, the free-energy gain is written as

$$
u_{d}=-M\left[\Delta \mu_{A}(1-c)+\Delta \mu_{B} c\right] .
$$




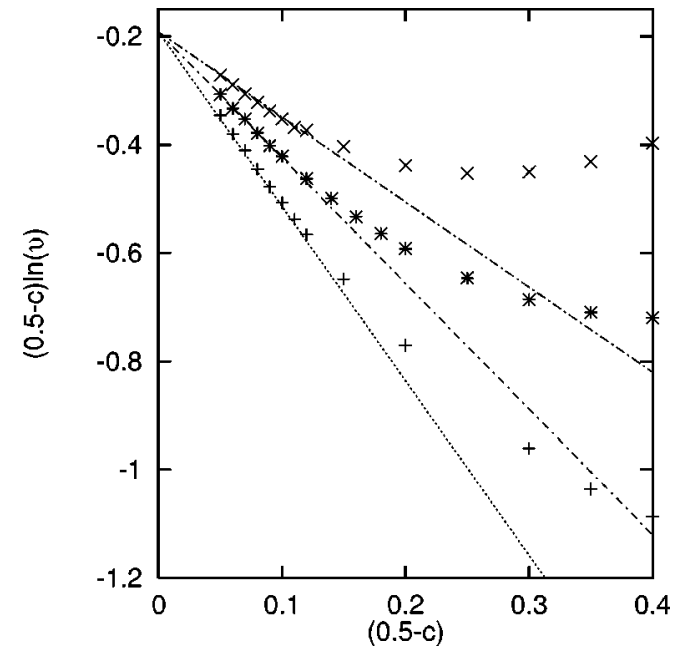

FIG. 10. Dependence of $(0.5-c) \ln (v)$, the normalized velocity, on the concentration difference $(0.5-c)$. The parameters are $N$ $=2000$ and $\epsilon=1.0 T_{A}(\times), 1.5 T_{A}(*)$, and $2.0 T_{A}(+)$, respectively.

The two terms on the RHS of the above equation represent the free-energy gain by solidification of $A$ and $B$ atoms, respectively. Allowing for the local concentration fluctuations, we can write the total free-energy gain as

$$
\begin{aligned}
u & =-\sum_{i=1}^{M}\left[\Delta \mu_{A}\left(1-c_{i}\right)+\Delta \mu_{B} c_{i}\right] \\
& \approx-M\left\{\Delta \mu_{A}[1-(c \pm \overline{\Delta c})]+\Delta \mu_{B}(c \pm \overline{\Delta c})\right\} \\
& =-M H \pm M \overline{\Delta c}\left(\Delta \mu_{B}-\Delta \mu_{A}\right) .
\end{aligned}
$$

Here $\overline{\Delta c}$ is the concentration fluctuation, $\overline{\Delta c}=\sqrt{\left\langle(\Delta c)^{2}\right\rangle}$, and is estimated as follows. Since the probability $p(M, m)$ of finding $m B$ atoms in the $M$ sites obeys the binomial distribution

$$
p(M, m)=\frac{M !}{m !(M-m) !} c^{m}(1-c)^{M-m},
$$

the mean value of $m$ is $\langle m\rangle=M c$ and its mean-square fluctuation is

$$
\left\langle(m-M c)^{2}\right\rangle=M c(1-c)
$$

Then

$$
\overline{\Delta c}=\sqrt{\left\langle\left(\frac{m}{M}-c\right)^{2}\right\rangle}=\sqrt{\frac{c(1-c)}{M}} .
$$

Substituting the expressions (60) into formula (57) and using the parameters $L_{A} / T_{A}=L_{B} / T_{B}=1, T_{A}=0.9, T_{B}=0.1$, and $T$ $=0.5$, the free-energy gain is expressed as follows:

$$
u=-0.8 M(0.5-c) \pm 0.8 \sqrt{M} \sqrt{c(1-c)} .
$$

Notifying that $M=h l$, the first term on the RHS corresponds to the bulk driving energy $u_{d}$, Eq. (51), and the second term corresponds to the random field contribution $u_{r}$, Eq. (49). Then the driving field is identified as

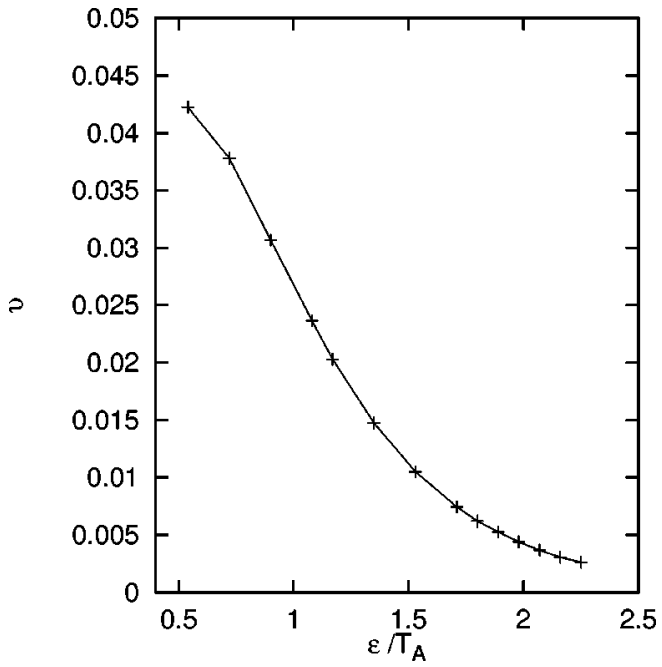

FIG. 11. Dependence of interface velocity $v$ on the bond energy $\epsilon$. The parameters are $N=2000, T=0.5, c=0.4$. Simulation is performed up to the time $10^{5}$.

$$
H=0.8(0.5-c)
$$

and the strength of the random field as

$$
\Delta=0.8 \sqrt{c(1-c)} .
$$

At the mean concentration, $c=0.5$, the driving force $H$ becomes zero and the strength of the random force $\Delta$ becomes 0.4. From the expressions (62) and (48), we obtain the prefactor $b$ as 0.8 . From $\Delta=0.4, b=0.8$, and the estimate $d \Delta^{2} / b \approx 0.096$ obtained by the simulation, $d$ is determined as 0.48 , of order unity as expected.

It is worth mentioning that the driving force $H$ used here is twice that of $\bar{f}$ used in the RFI model (16): $H=2 \bar{f}$. The factor 2 results from the fact that the energy gain is $2 u_{d}$ when atoms change states from liquid to solid, or in terms of the RFI model, spins flip from downwards $\left(s_{i}=-1\right)$ to upwards $\left(s_{i}=+1\right)$.

\section{E. The effect of the bond energy on the velocity}

Obviously, the larger the bond energy is, the more difficult it is for the interface to move. Figures 8 and 10 clearly show this tendency. For a systematic study of the $\epsilon$ dependence of the velocity, we fix the concentration $c=0.4$ and vary $\epsilon$. Figure 11 shows the result for the system size $N$ $=2000$ and $T=0.5$. From the figure we can see that the velocity decays with increasing the energy $\epsilon$. Generally we cannot formulate the velocity as a function of $\epsilon$, but for large energy things become simple. For large energy the interface is almost flat, but since the system size is large enough, still a few kinks are always excited thermally. The density of those thermally excited kinks is proportional to $\exp (-\epsilon / T)$ and they act as a solidification center. With increasing energy the difficulty of creating a kink increases as well and therefore fewer kinks exist. Because the propagation of a kink along the interface is much faster than creating it, we can safely predict that the velocity decays in the same manner as the number of kinks: $v \sim \exp (-\epsilon / T)$. Figure 12 shows the data of Fig. 11 in a semilogarithmic way. The data in the 


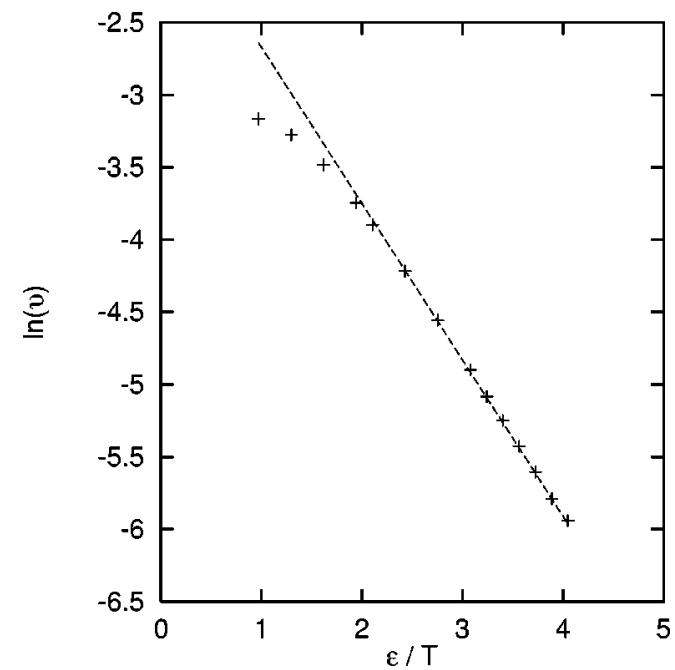

FIG. 12. Logarithm of the velocity $\ln (v)$ versus the bond energy $\epsilon / T$. The fitting line has a slope around 1.06.

large $\epsilon / T$ range can be fitted by a straight line with slope around unity, which agrees quite well with the prediction. This argument is not applicable for small $\epsilon$ because multikinks exist on the interface.

Combining the above argument with the result Eq. (54) in the preceding subsection, we get the velocity of the solidification front for an infinite system with large bond energy under very small driving force ( $c$ being in the vicinity of 0.5 ) as

$$
v=a e^{-\epsilon / T} e^{-d \Delta^{2} / H T}
$$

From Fig. 10 we estimate the constant value of $a$ around 1.5 and $d \approx 0.5$ as estimated in Sec. VD.

Some comments may be necessary concerning a small system. As is shown in the Appendix, explicitly the velocities of small systems are proportional to $\exp (-2 \epsilon / T)$ for $\epsilon_{s}$ $=\epsilon_{l}=\epsilon$. This is due to the fact that the creation of an initial nucleus is required in these small systems, because there exists usually no kinks at all at sufficiently large bond energy and low enough temperature.

\section{F. Very small bond energy regime}

For very small but nonzero bond energy $\left(\epsilon / T_{A} \ll 1\right)$, namely the jump regime (a) of the two-phase region in Fig. 6 , the interface is pinned and therefore does not have a steady-state velocity. The fact that the exponent $\nu$ increases from the value 0.51 of a $1 \mathrm{D}$ system to 1.0 when $\epsilon$ is around $0.1 T_{A}$ reveals that the $2 \mathrm{D}$ effect on the system gets stronger as $\epsilon$ increases. Pinning for small bond energy $\epsilon$ can be understood by the following picture: treat the very small energy $\epsilon$ as a perturbation for the zero- $\epsilon$ case. In the absence of the bond energy, there exists no correlation between neighboring atomic columns in the 2D system and it becomes simply a pile of 1D chains. Once an atom is pinned, it will stay in the pinned state until becoming excited into the unpinned state by the external driving force $H$ and it gets no help from its neighbors. Therefore, the interface is rather corrugated and interspersed with many ditches with a $B$ atom sitting at the bottom. The dynamic exponent $\nu$ can be approximately expressed as

$$
\nu \approx \frac{H}{H_{c}}=\frac{C_{0}-c}{C_{0}-C_{s}} .
$$

Here $H_{c}$ is the critical driving force which separates the moving interface (when $H>H_{c}$ ) from the pinned interface (when $H<H_{c}$ ) for a one-dimensional diffusionless disordered alloy system. The above formula (65) shows a linear dependence of $\nu$ on $c$. The suitability of this approximation (65) can be verified by Fig. 2, where the solid line shows an approximately linear decrease of $\nu$ from 1 at $c=C_{s}$ to 0 at $c=C_{0}$ in the solid side of the two-phase region.

As the small bond energy is introduced, the atoms in different columns start to affect each other. The bond energy plays the role of an extra driving force which always tries to make the pinned atomic column catch up with its two neighboring advancing columns. As a result, when the pinned atom solidifies, the system gets energy gain not only from the driving force $b\left(C_{0}-c\right)$ but also from the bond $2 \epsilon$ with its two neighboring atoms. According to Eq. (65), $\nu$ can be now approximately written as

$$
\nu \approx \frac{H}{H_{c}}=\frac{b\left(C_{0}-c\right)+2 \epsilon / T_{A}}{b\left(C_{0}-C_{s}\right)} .
$$

This formula shows a linear dependence of $\nu$ on $\epsilon$. Roughly speaking, this is what we have seen in the jump regime of Fig. 6. Because of the extra driving force $2 \epsilon$, the real external driving force $b\left(C_{0}-c\right)$ required to keep the interface steadily moving becomes smaller than that $\left[b\left(C_{0}-C_{s}\right)\right]$ in the zero- $\epsilon$ case. In other words, even for $b\left(C_{0}-c\right)<H_{c}$ or $c>C_{s}$, it is still possible that the interface moves steadily provided that $\epsilon$ is bigger than a critical value $\epsilon^{*}$, which satisfies the condition $\nu\left(\epsilon^{*}\right)=1$. In the case studied here $\left(c=0.4, b=0.8, C_{s}=0.31\right.$, and $\left.C_{0}=0.5\right), \epsilon^{*}$ is about $0.04 T_{A}$ from the formula (66) by letting $\nu=1$. The value of $\epsilon^{*}$ is measured from the simulation at around $0.1 T_{A}$ (see Fig. 6). This means that the interface gets pinned when $\epsilon<\epsilon^{*}$ but unpinned when $\epsilon>\epsilon^{*}$ in the two-phase region of the equilibrium phase diagram for very small $\epsilon$. In addition, $\epsilon^{*}$ varies with the concentration and the temperature. Note that this argument applies only to the very small $\epsilon^{*}$ case where such an interface structure of a single pinned atomic column surrounded by two much advanced columns could exist and $\epsilon^{*}$ can be considered as a perturbation.

\section{G. Correlation function}

In Sec. V C we have observed that the interface displacement for a finite system crosses over from the depinned to the pinned state as time proceeds. When does the system undergo a depinning-pinning transition? What is the mechanism that leads to the transition? To answer these questions, we must discuss the development of correlations in the system. Different sites of the interface are not completely independent, but their height and kink structure depends upon the heights of the neighboring sites. Figure 13 shows the spatial correlation function

$$
G(x, t)=\left\langle h\left(x^{\prime}, t\right)^{2}\right\rangle-\left\langle h\left(x^{\prime}, t\right) h\left(x^{\prime}+x, t\right)\right\rangle
$$

at various times. 


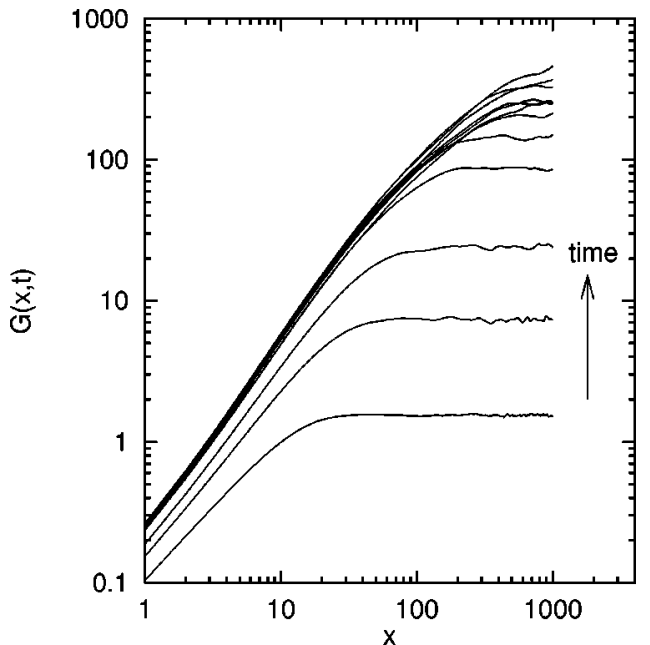

FIG. 13. Time variation of the space correlation function up to the time $10^{5}$. The simulation parameters are $N=2000, c=0.4, T$ $=0.5, \epsilon=1.5 T_{A}$. Data are averaged for 50 independent runs.

Here $x^{\prime}$ and $x+x^{\prime}$ are two different lattice sites, and $\langle\cdots\rangle$ means the average over all sites $x^{\prime}$ keeping the separation $x$ fixed. It is apparent in Fig. 13 that at a fixed time the correlation function increases with the separation $x$ but saturates for $x$ larger than the correlation length $\xi_{/ /}$: The correlation extends up to $\xi_{/ /}$but two points on the interface separated by more than $\xi_{/ /}$are uncorrelated. As time proceeds, the correlation length $\xi_{/ /}$increases with time, as is shown in Fig. 14. The fitting curve $\xi_{/ /} \sim t^{1 / 3}$ is drawn just as a guide to the eyes.

For $x \gg \xi_{/ /}$the correlation of the interface Eq. (67) at two points is lost and the average $\left\langle h\left(x^{\prime}\right) h\left(x+x^{\prime}\right)\right\rangle$ can be decoupled as

$$
\lim _{x \rightarrow \infty} G(x, t)=\left\langle h(t)^{2}\right\rangle-\langle h(t)\rangle^{2}=\sigma^{2}(t) .
$$

The saturation value of the correlation function in the limit of large separation $x$ is equal to the variance $\sigma^{2}(t)$ of the interface position $h(t)$. Note, however, that the width of the interface at long times scales with the system size is

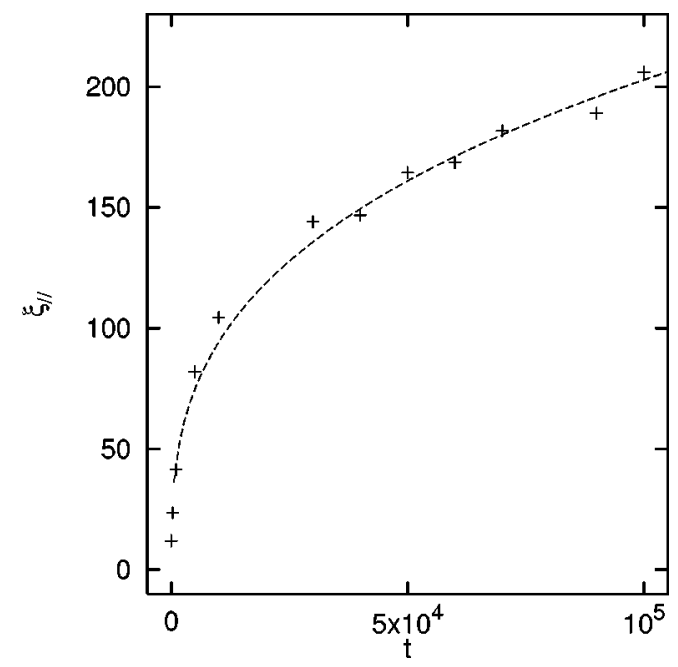

FIG. 14. Correlation length $\xi_{/ /}$versus the time $t$. The simulation is performed with the system size $N=2000, \epsilon=1.5 T_{A}, T=0.5$, and $c=0.4$. The curve $\xi_{/ /} \sim t^{1 / 3}$ is just a guide for the eyes.

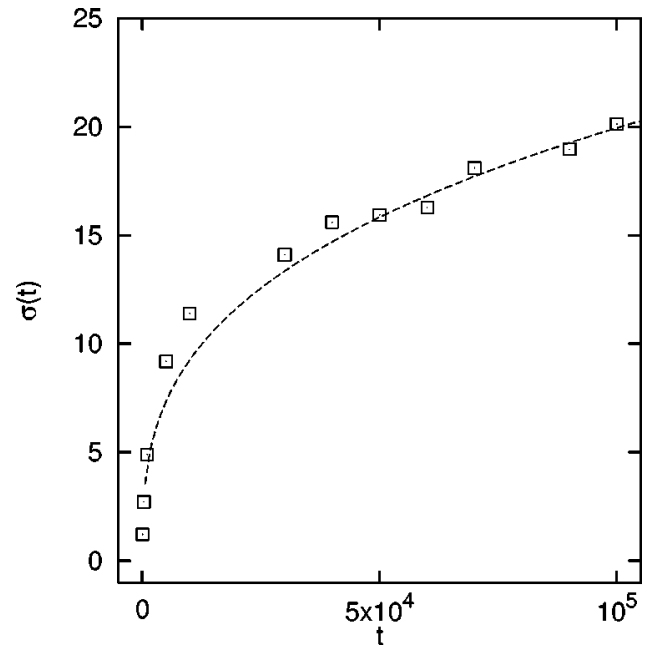

FIG. 15. Time evolution of the width of the interface $\sigma(t)$. The curve $\sigma(t) \sim t^{1 / 3}$ is just to guide the eyes.

$\sigma \sim N^{0.69}$, as can be read off Fig. 13. Figure 15 shows the interface width $\sigma(t)$ as a function of time $t$. At time $10^{5}$, $\sigma(t)$ is about 20 , which is at least less than $1 / 15$ of the width of the interface in a 1D system with the same concentration $(c=0.4)$ [4]. The thin interface width in the 2D system is due to the surface tension which tends to keep the interface as flat as possible.

The pinning-depinning crossover phenomena can be understood in terms of the correlation length and the finite size of the system. From the simulation of a rather large system $N=2000$, we know how the correlation develops as time proceeds, as shown in Fig. 14. For systems with small sizes the correlation should extend similarly. Therefore the time dependence of the velocity can be related to the $\xi_{/ /}$dependence of the velocity, as shown in Fig. 16. It contains the information of four different system sizes. At the very beginning the interface is not affected by the system size and all systems behave in the same way. This corresponds to the common slope $(\sim 0.0263)$ of the initial part of the curves in

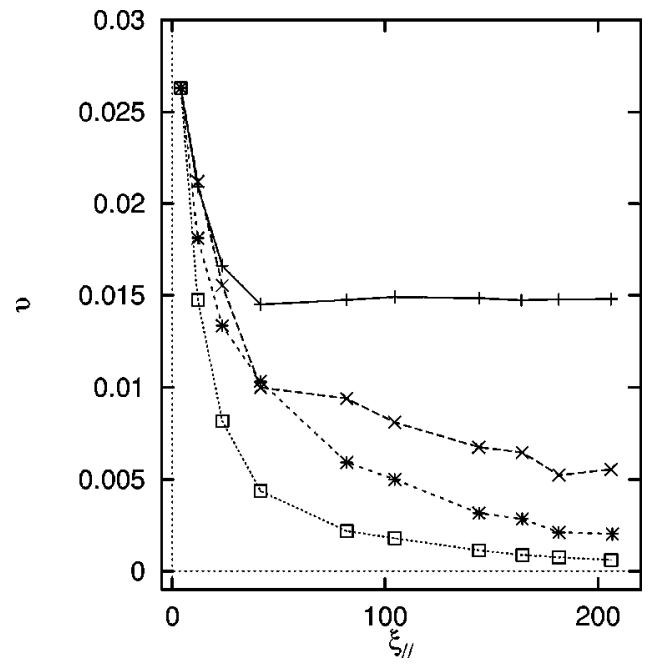

FIG. 16. The velocity $v$ of the interface versus the correlation length $\xi_{/ /}$. The system sizes are $N=10,25,40$, and 2000 from the bottom to the top, respectively. 
Fig. 5. As time passes and when the correlation length approaches the system size, the interface starts to slow down and eventually the velocity becomes zero. This crossover from depinning to pinning takes place later if the system size becomes larger. For a very large system $(N=2000)$, $\xi_{/ /}$remains much smaller than $N$ and the interface stays in the depinned state with a steady-state velocity.

\section{CONCLUSION}

We have studied the diffusionless solid-liquid phase transformation in a two-dimensional binary alloy by observing the motion of the solid-liquid interface. Monte Carlo simulations show that in the one-phase region the crystal grows with a steady-state velocity, and its bond energy dependence and concentration dependence can be decoupled as $v(\boldsymbol{\epsilon}, c)=f_{1}(\boldsymbol{\epsilon}) f_{2}(c)$ when the bond energy $\boldsymbol{\epsilon}$ is not very small. As far as we know, there is no theory on this $\epsilon$ and $c$ dependence of $v$ so far.

In the two-phase region, the interface shows a complicated behavior depending upon the system size $N$, the bond energy $\epsilon$, and the simulation time $t$. For a finite-sized system under finite simulation time (up to $t=10^{5}-10^{6}$ ), the growth exponent $\nu$ depends strongly on $\epsilon$ (see Fig. 6):

$$
\begin{gathered}
\nu<1 \text { for } \epsilon<0.1 T_{A}, \\
\nu=1 \text { for } 0.1 T_{A}<\epsilon<\epsilon_{c}(N), \\
\nu<1 \text { for } \epsilon>\epsilon_{c}(N), \\
\nu=\nu(1 D) \text { for } \epsilon \rightarrow \infty .
\end{gathered}
$$

Unlike the one-dimensional system, the two-dimensional system can realize a steady growth also in the two-phase region $\left[0.1 T_{A}<\epsilon<\epsilon_{c}(N)\right]$. However, when $\boldsymbol{\epsilon}>\boldsymbol{\epsilon}_{c}(N)$, the interface becomes pinned such that the spatial disorder of the alloy leads to a decay of the interface velocity. In the large energy limit $(\epsilon \rightarrow \infty)$, theoretical analysis and computer simulations show that the exponent $\nu$ has the same value as its one-dimensional counterpart $\nu(1 \mathrm{D})$.

A peculiar result occurs for small but nonzero bond energies, less than about $0.1 T_{A}$, as discussed in Sec. V F. Here pinning occurs as would be the case for vanishing bond energy but the exponent $\nu$ is increased above the value of the one-dimensional case $(\nu \simeq 0.51)$. Therefore both for very large and very small bond energy in a finite system, the interface will be pinned. Note, however, that the latter result applies only to models of SOS type and probably not to systems with overhangs.

For a very large system $(N \rightarrow \infty)$ with a finite bond energy, the simulation reveals that the interface always moves steadily within a finite simulation time that means for the critical bond energy $\epsilon_{c}(N \rightarrow \infty) \rightarrow \infty$. This corresponds to the thermal creep motion. We found that the influences of the bond energy $\epsilon$ and the driving force $H$ on the velocity $v$ of the interface growth are decoupled as indicated in Eq. (64). The velocity $v$ decays exponentially with $\epsilon$ and $H^{-1}$ in the vicinity of zero $H$ and for sufficiently large $\epsilon$.

In addition, we have also studied the development of correlations along the interface and their relevance to finite-size effects on the interface dynamics. The correlation length $\xi_{/ /}$ along the interface increases very slowly with time, Fig. 14. If it reaches the system size $N$, the whole interface becomes correlated and the interface asymptotically behaves like the one in a one-dimensional system, but as long as the system size is larger than $\xi_{/ /}$the thermal creep wins over the pinning force. Thus for the system studied here the limits $N \rightarrow \infty$ and $t \rightarrow \infty$ are not interchangeable.

\section{ACKNOWLEDGMENTS}

Y.S. and H.M-K. gratefully acknowledge support from the Germany-Japan exchange program of the Japanese Society of Promotion of Science and the Deutsche Forschungsgemeinschaft. We also appreciate support by Volkswagen Foundation and the Russian Fund for Fundamental Research (Project No. 98-02-16249).

\section{APPENDIX}

Here we give closed-form expressions for the elementary layer-jump frequencies $\Omega_{+,-}$introduced in Sec. IV, valid for small values of the system size $N$. For small enough $N$ we can analytically solve Eq. (35). For infinitely large bonding energies, $\epsilon_{s}$ and $\epsilon_{l}$, the transition frequencies have large differences $\omega_{ \pm i}(1)=\omega_{ \pm i} \exp \left(\epsilon_{l(s)} / T\right) \gg \omega_{ \pm i} \gg \omega_{ \pm i}(3)$ $=\omega_{ \pm i} \exp \left(-\epsilon_{l(s)} / T\right)$. Then the closed analytical form of $\Omega_{+}$ introduced in Sec. IV is obtained for $N=2,3$, and 4 from Eqs. (29), (35), and (36). For $N=2$,

$$
\Omega_{+}=\omega_{+1} \omega_{+2}\left\{\frac{1}{\omega_{+2}(1)+\omega_{-1}(1)}+\frac{1}{\omega_{+1}(1)+\omega_{-2}(1)}\right\} .
$$

For $N=3$,

$$
\begin{aligned}
\Omega_{+}= & \frac{\exp \left[-\left(\epsilon_{s}+\epsilon_{l}\right) / T\right]}{\omega_{-1} \omega_{-2} \omega_{-3}}\left[\omega_{+1} \omega_{-1}\left(\omega_{+2} \omega_{-3}+\omega_{-2} \omega_{+3}\right)\right. \\
& +\omega_{+2} \omega_{-2}\left(\omega_{+1} \omega_{-3}+\omega_{-1} \omega_{+3}\right) \\
& \left.+\omega_{+3} \omega_{-3}\left(\omega_{+1} \omega_{-2}+\omega_{-1} \omega_{+2}\right)\right]
\end{aligned}
$$

For $N=4$,

$$
\begin{aligned}
& \Omega_{+}= \frac{\exp \left[-\left(\epsilon_{s}+\epsilon_{l}\right) / T\right]}{4} \\
& \prod_{i=1}^{4} \omega_{-i} \\
& \times \sum_{i=1}^{4} \omega_{+i} \omega_{+(i+1)} \omega_{-(i+2)} \omega_{-(i+3)} \\
& \times \frac{\left(\omega_{+(i+2)}+\omega_{+(i+3)}\right)\left(\omega_{-i}+\omega_{-(i+1)}\right)}{\omega_{+(i+2)}+\omega_{+(i+3)}+\omega_{-i}+\omega_{-(i+1)}} .
\end{aligned}
$$

The expressions for $\Omega_{-}$can be obtained from the corresponding ones for $\Omega_{+}$by replacing $\omega_{+j}$ with $\omega_{-j}$ and vice versa, where $j$ stands here for any index in Eq. (A3).

The solidification velocity $v_{+}$can also be calculated explicitly as follows.

For $N=1$, 


$$
v_{+}=e^{-\epsilon_{l} / T}\left(1-S_{+} e^{\left(\epsilon_{l}-\epsilon_{s}\right) / T}\right) /\left(\frac{1-c}{\omega_{+A}}+\frac{c}{\omega_{+B}}\right)
$$

For $N=2$,

$$
\begin{aligned}
v_{+}= & \left(1-S_{+}^{2}\right) /\left\{\frac{(1-c)^{2}}{2 \omega_{+A}^{2}}\left(\omega_{+A} e^{\epsilon_{l} / T}+\omega_{-A} e^{\epsilon_{s} / T}\right)\right. \\
& \left.+\frac{2 c(1-c)\left(\omega_{+A} e^{\epsilon_{l} / T}+\omega_{-B} e^{\epsilon_{s} / T}\right)\left(\omega_{+B} e^{\epsilon_{l} / T}+\omega_{-A} e^{\epsilon_{s} / T}\right)}{\omega_{+A} \omega_{+B}\left(\omega_{+A}+\omega_{+B}\right) e^{\epsilon_{l} / T}+\left(\omega_{-A}+\omega_{-B}\right) e^{\epsilon_{s} / T}}+\frac{c^{2}}{2 \omega_{+B}^{2}}\left(\omega_{+B} e^{\epsilon_{l} / T}+\omega_{-B} e^{\epsilon_{s} / T}\right)\right\} .
\end{aligned}
$$

For $N=3$,

$$
\begin{aligned}
v_{+}= & e^{-\left(\epsilon_{s}+\epsilon_{l}\right) / T}\left(1-S_{+}^{3}\right) /\left\{\frac{(1-c)^{3} \omega_{-A}}{6 \omega_{+A}^{2}}\right. \\
& \left.+\frac{3(1-c)^{2} c \omega_{-A} \omega_{-B}}{2 \omega_{+A}\left(\omega_{+A} \omega_{-B}+\omega_{+B} \omega_{-A}+\omega_{+B} \omega_{-B}\right)}+\frac{3(1-c) c^{2} \omega_{-A} \omega_{-B}}{2 \omega_{+B}\left(\omega_{+A} \omega_{-B}+\omega_{+B} \omega_{-A}+\omega_{+A} \omega_{-A}\right)}+\frac{c^{3} \omega_{-B}}{6 \omega_{+B}^{2}}\right\} .
\end{aligned}
$$

For $N=4$,

$$
\begin{aligned}
v_{+}= & e^{-\left(\epsilon_{s}+\epsilon_{l}\right) / T}\left(1-S_{+}^{4}\right) /\left\{(1-c)^{4} \frac{\omega_{-A}\left(\omega_{+A}+\omega_{-A}\right)}{8 \omega_{+A}^{3}}+(1-c)^{3} c \frac{\omega_{-A} \omega_{-B}}{\omega_{+A}^{2}}\right. \\
& \times\left(\frac{\left(\omega_{+A}+\omega_{+B}\right) \omega_{-B}}{2 \omega_{-A}+\omega_{+A}+\omega_{+B}}+\frac{\left(\omega_{-A}+\omega_{-B}\right) \omega_{+B}}{2 \omega_{+A}+\omega_{-A}+\omega_{-B}}\right)^{-1}+2 c^{2}(1-c)^{2} \frac{\omega_{-A} \omega_{-B}}{\omega_{+A} \omega_{+B}}\left(\frac{\omega_{+A} \omega_{-B}}{\omega_{-A}+\omega_{+B}}\right. \\
& \left.+\frac{\left(\omega_{+A}+\omega_{+B}\right)\left(\omega_{-A}+\omega_{-B}\right)}{\omega_{+A}+\omega_{-A}+\omega_{+B}+\omega_{-B}}+\frac{\omega_{-A} \omega_{+B}}{\omega_{+A}+\omega_{-B}}\right)^{-1}+\frac{c^{2}(1-c)^{2}}{2} \frac{\omega_{-A} \omega_{-B}\left(\omega_{+A}+\omega_{-A}+\omega_{+B}+\omega_{-B}\right)}{\omega_{+A} \omega_{+B}\left(\omega_{+A}+\omega_{+B}\right)\left(\omega_{-A}+\omega_{-B}\right)} \\
& \left.+(1-c) c^{3} \frac{\omega_{-A} \omega_{-B}}{\omega_{+B}^{2}}\left(\frac{\left(\omega_{+A}+\omega_{+B}\right) \omega_{-A}}{\omega_{+A}+\omega_{+B}+2 \omega_{-B}}+\frac{\left(\omega_{-A}+\omega_{-B}\right) \omega_{+A}}{\omega_{-A}+2 \omega_{+B}+\omega_{-B}}\right)^{-1}+c^{4} \frac{\omega_{-B}\left(\omega_{+B}+\omega_{-B}\right)}{8 \omega_{+B}^{3}}\right\}
\end{aligned}
$$

Here $S_{+}$is defined in Eq. (41). We should note that the result for $N=1$ in Eq. (A4) differs from the usual result, Eq. (3), for the pure one-dimensional system by the presence of the exponential factors. This difference is due to the periodic boundary condition.

The analytical result of the velocity $v_{+}$is compared with numerical simulation results explicitly for the case with system size $N=4$ in Sec. IV B. We have also checked the results for smaller systems and found good agreement, within $1 \%$.

In Sec. IV, we found that the 2D system with a large bond energy is effectively a one-dimensional system with the transition rates $\Omega_{+}$and $\Omega_{-}$. Then we can use some additional results for a real one-dimensional system. For example, following the procedure of Ref. [2] we can derive the expression $W\left(\left\{X^{(n)}\right\}\right)$ for the probability of finding a configuration $\left\{X^{(n)}\right\} \equiv\left\{X_{1}^{(n)} \cdots X_{N}^{(n)}\right\}$ in the $n$th layer from the steadily growing interface:

$$
W\left(\left\{X^{(n)}\right\}\right)=\prod_{i=1}^{N} c_{X_{i}^{(n)}} \text { for } n \leqslant-1, \text { in the solid, }
$$

and

$$
W\left(\left\{X^{(n)}\right\}\right)=\prod_{i=1}^{N} c_{X_{i}^{(n)}}\left\{1+S_{+}^{N n}\left(\frac{\Omega_{-}\left(\left\{X^{(n)}\right\}\right)}{\Omega_{+}\left(\left\{X^{(n)}\right\}\right)}-1+\frac{1-S_{+}^{N}}{\Omega_{+}\left(\left\{X^{(n)}\right\}\right)\left\langle 1 / \Omega_{+}\right\rangle}\right)\right\} \quad \text { for } n \geqslant 0 \text {, in the liquid. }
$$

From Eq. (A8) one can calculate the average concentration of $B$ atoms, $c(n)$, in the $n$th layer from the interface, by fixing the configuration of one lattice site, say $i=1$, to a $B$ atom $\left(X_{1}^{(n)}=B\right)$, and by the summing $W\left(\left\{X^{(n)}\right\}\right)$ over all possible configurations $\left\{X^{(n)}\right\}=\left\{B, X_{2}^{(n)} \cdots X_{N}^{(n)}\right\}$. For example, when the initial concentration is $c=C_{S}$, where $S_{+}=1$ and the steady-state velocity $v_{+}=0, c(n)$ is obtained as

$$
c(n)= \begin{cases}C_{S} & \text { for } n \leqslant-1, \\ C_{L} & \text { for } n \geqslant 0\end{cases}
$$

Both phases have the equilibrium compositions, as one finds in the case of the real one-dimensional system [2]. 
[1] D. E. Temkin, J. Cryst. Growth 5, 193 (1969).

[2] D. E. Temkin, Dokl. Akad. Nauk SSSR 206, 27 (1972) [Sov. Math. Dokl. 13, 1172 (1972)].

[3] D. E. Temkin, Kristallografiya 17, 77 (1972) [Sov. Phys. Crystallogr. 17, 60 (1972)].

[4] Y. Saito, N. Tanabe, and D. Temkin, Phys. Rev. E 48, 2028 (1993).

[5] X. Feng, E. Brener, D. Temkin, Y. Saito, and H. MüllerKrumbhaar, Euro. Phys. J. B (to be published).

[6] J. Bernasconi and W. R. Schneider, J. Phys. A 15, L729 (1982).

[7] B. Derrida and Y. Pomeau, Phys. Rev. Lett. 48, 627 (1982).

[8] B. Derrida, J. Stat. Phys. 31, 433 (1983).

[9] C. Asulangul, M. Barthelemy, N. Pottier, and D. Saint-James, J. Stat. Phys. 59, 11 (1990).

[10] J. P. Bouchaud and A. Georges, Phys. Rep. 195, 127 (1990).
[11] J. P. Bouchaud, A. Comtet, A. George, and P. Le Doussal, Ann. Phys. (N.Y.) 201, 285 (1990).

[12] Ya. G. Sinai, in Mathematical Problems in Theoretical Physics, edited by R. Schneider, R. Seiler, and P. Uhlenbrock, Lecture Notes in Physics Vol. 153 (Springer, Berlin, 1982), p. 12.

[13] D. E. Temkin, Kristallografiya 19, 476 (1974) [Sov. Phys. Crystallogr. 19, 291 (1974)].

[14] K. A. Jackson, G. H. Gilmer, D. E. Temkin, J. D. Weinberg, and K. Beatty, J. Cryst. Growth 128, 127 (1993).

[15] V. V. Voronkov, Kristallografiya, 19, 475 (1974) [Sov. Phys. Crystallogr. 19, 296 (1974)].

[16] T. Nattermann, Spin Glasses and Random Fields, edited by A. P. Young (World Scientific, Singapore, 1998).

[17] L. B. Ioffe and V. M. Vinokur, J. Phys. C 20, 6149 (1987).

[18] V. M. Vinokur, Physica D 107, 411 (1997).

[19] Y. Imry and S. K. Ma, Phys. Rev. Lett. 35, 1399 (1975). 